Mississippi State University

Scholars Junction

$5-3-2019$

\title{
Efficacy of herbicide spray droplet size, flooding period, and seed burial depth on Palmer amaranth (Amaranthus palmeri S. Wats.) control
}

Lucas Xavier Franca

Follow this and additional works at: https://scholarsjunction.msstate.edu/td

\section{Recommended Citation}

Franca, Lucas Xavier, "Efficacy of herbicide spray droplet size, flooding period, and seed burial depth on Palmer amaranth (Amaranthus palmeri S. Wats.) control" (2019). Theses and Dissertations. 5072. https://scholarsjunction.msstate.edu/td/5072

This Dissertation - Open Access is brought to you for free and open access by the Theses and Dissertations at Scholars Junction. It has been accepted for inclusion in Theses and Dissertations by an authorized administrator of Scholars Junction. For more information, please contact scholcomm@msstate.libanswers.com. 
Efficacy of herbicide spray droplet size, flooding period, and seed burial depth on Palmer amaranth (Amaranthus palmeri S. Wats.) control

By

Lucas Xavier Franca

\begin{abstract}
A Dissertation
Submitted to the Faculty of Mississippi State University in Partial Fulfillment of the Requirements for the Degree of Doctor of Philosophy in Weed Science in the Department of Plant and Soil Sciences
\end{abstract}

Mississippi State, Mississippi

May 2019 
Copyright by

Lucas Xavier Franca

2019 
Efficacy of herbicide spray droplet size, flooding period, and seed burial depth on Palmer amaranth (Amaranthus palmeri S. Wats.) control

By

Lucas Xavier Franca

Approved:

Darrin M. Dodds

(Major Professor)
Angus L. Catchot Jr.

(Minor Professor)

Daniel B. Reynolds

(Committee Member)

Jason A. Bond

(Committee Member)

Daniel G. Peterson

(Committee Member)

J. Anthony Mills

(Committee Member)

Michael S. Cox

(Graduate Coordinator)

George M. Hopper

Dean

College of Agriculture and Life Sciences 
Name: Lucas Xavier Franca

Date of Degree: May 3, 2019

Institution: Mississippi State University

Major Field: Weed Science

Major Professor: Darrin M. Dodds

Title of Study: Efficacy of herbicide spray droplet size, flooding period, and seed burial depth on Palmer amaranth (Amaranthus palmeri S. Wats.) control

Pages in Study 89

Candidate for Degree of Doctor of Philosophy

The continued spread of Palmer amaranth (Amaranthus palmeri S. Wats.) throughout the southern and midwestern United States is a result of herbicide-resistant populations. Besides being the most troublesome weed specie in several agronomic crops, Palmer amaranth is also host to economically important pests such as tarnished plant bug (Lygus lineolaris Palisot de Beauvois). Pesticide application methodology that maximizes efficacy while reducing selection pressure is needed to combat herbicideresistant Palmer amaranth. Pulse width modulation (PWM) sprayers are used for pesticide application with the goal of maintaining product efficacy while mitigating spray drift. Additionally, alternative off-season weed management practices such as flooding could be adopted to optimize soil seedbank depletion. Therefore, evaluation of spray droplet size and flooding period on Palmer amaranth control and seed germination was conducted.

The objectives of this research were to: (1) evaluate the influence of spray droplet size on lactofen and acifluorfen efficacy on Palmer amaranth using a PWM sprayer, (2) develop prediction models to determine spray droplet size that provides the greatest level 
of Palmer amaranth control, (3) evaluate the impact of flooding period and seed burial depth on Palmer amaranth seed germination in different soil textures, and (4) analyze the impact of nitrogen fertilizer application rate on the attractiveness of Palmer amaranth to tarnished plant bug.

Results show that spray droplet size does not affect lactofen efficacy on Palmer amaranth, thus, coarser spray droplets are recommended to increase spray drift mitigation efforts. In contrast, acifluorfen applied with $300 \mu \mathrm{m}$ (medium) spray droplets provided the greatest Palmer amaranth control. Furthermore, prediction models indicated that greater model accuracy was obtained when adopting a location-specific weed management approach. Flooding periods of 3, 4, and 5 months reduced Palmer amaranth seed germination across burial depths and soil textures. Therefore, fall-winter flooding may be adopted as an effective practice for soil seedbank depletion. Results also demonstrated that nitrogen fertilizer application rate does not consistently impact Palmer amaranth attractiveness to tarnished plant bug. 


\section{DEDICATION}

I would like to dedicate this achievement to my Avó (grandmother), Marivalda Clara da Silva, and Bisavó (great-grandmother) Enedina Ferreira Galvão, who passed away during my studies abroad. Thank you very much for your support, prayers, and endless conversations. You have taught me to be resilient and always trust in God, especially in difficult times. I will continue to work hard to make you proud until the end of my days. I would also like to dedicate this dissertation to my parents, Renato Paiva França and Lourdes Maria Xavier, and to my sister, Barbara Xavier França. Thank you for the endless love, care, dedication, and support. Without you in my life, I would have not been able to accomplish this degree. In addition, I dedicate this achievement to my

girlfriend, Saskia P. Sauter. Your love, encouragement, dedication, and daily support made this possible. There are not words to describe how thankful I am for having you in my life. I cannot wait to start a new chapter in our lives. Muito obrigado a todos vocês! 


\section{ACKNOWLEDGEMENTS}

I would like to take this opportunity to sincerely thank all those individuals who have assisted, supported, and influenced me throughout the course of my Ph.D. at Mississippi State University. I would like to thank my major professor, Dr. Darrin Dodds for the mentorship, guidance, and for providing me all the resources to develop and become a better professional. Thank you for pushing me when I needed, and for the opportunity to attend a multitude of scientific conferences, meetings, trainings, and courses. The knowledge and practical skills obtained throughout my Ph.D. will be with me for the rest of my life. I would also like to thank the other members of my graduate committee, Drs. Daniel Reynolds, Angus Catchot, Jason Bond, Daniel Peterson, and Anthony Mills for their guidance, assistance, technical input, and for always being available to exchange ideas and thoughts. Further, I would like to thank Dr. Anthony Mills for choosing me to be one of the recipients of the prestigious Will D. Carpenter Distinguished Field Scientist Assistantship. The professional exposure, networking, and practical training provided by this opportunity will add immeasurable value to my future career.

I also would like to thank my past and current fellow graduate students, student workers, and staff members who have contributed and helped throughout my Ph.D. including: Michael T. Plumblee, Chase A. Samples, Bradley (Nunchuck) Wilson, Savana S. Davis, Jacob McNeal, and Bradley (Lurch) Norris. Thank you for your help whether it 
be planting trials, spraying plots, counting seed, rating experiments, or reviewing papers and abstracts. Additionally, I would like to extend my gratitude to Dr. Jonathan Huff and Darla Huff for their endless support, love, and everlasting friendship. 


\section{TABLE OF CONTENTS}

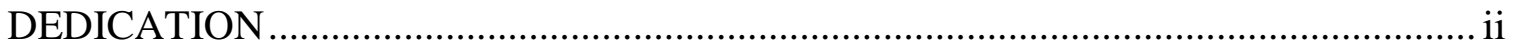

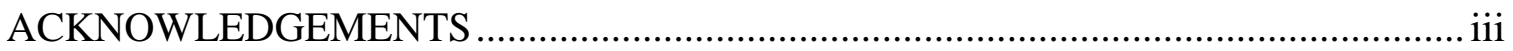

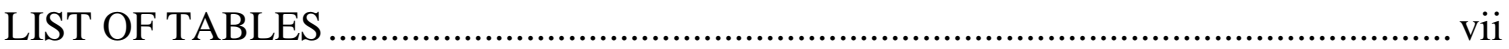

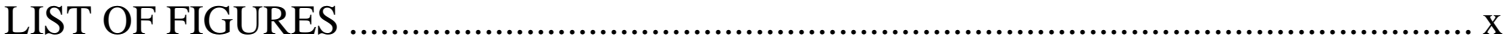

\section{CHAPTER}

I. DROPLET SIZE IMPACT ON LACTOFEN AND ACIFLUORFEN EFFICACY FOR PALMER AMANRATH (AMARANTHUS PALMERI S. WATS.) CONTROL ......................................................

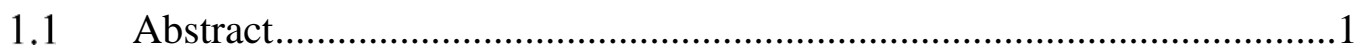

$1.2 \quad$ Introduction .................................................................................

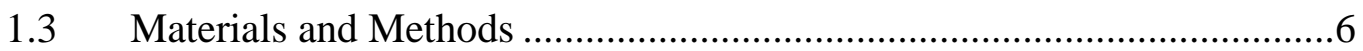

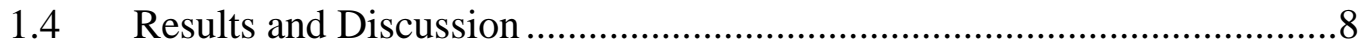

1.4.1 Palmer amaranth control with lactofen..............................................9

1.4.2 Palmer amaranth control with acifluorfen ........................................10

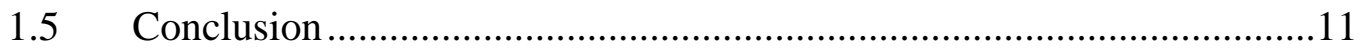

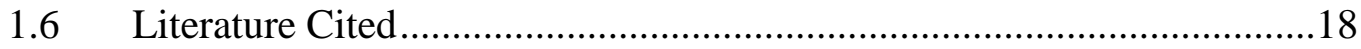

II. PALMER AMARANTH (AMARANTHUS PALMERI S. WATS.) CONTROL USING VARIOUS DROPLET SIZES OF ACIFLUORFEN

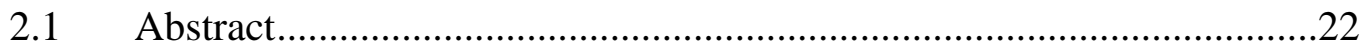

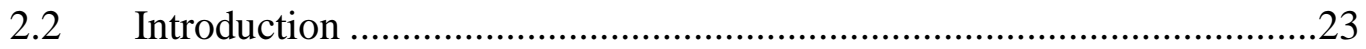

2.3 Materials and Methods ............................................................................

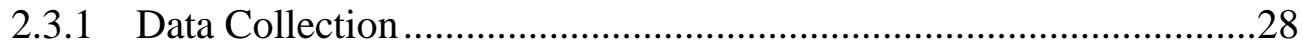

2.3.2 Statistical Analysis .......................................................................28 


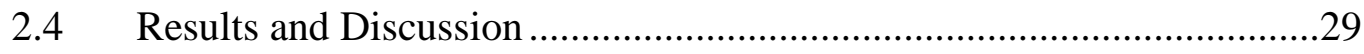

2.4.1 Acifluorfen location-specific analysis ..........................................31

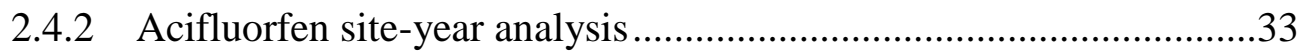

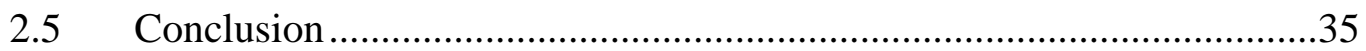

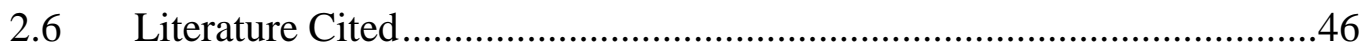

III. INFLUENCE OF FLOODING PERIOD AND SEED BURIAL DEPTH ON PALMER AMARANTH (AMARANTHUS PALMERI S. WATS.) SEED GERMINATION ......................................................49

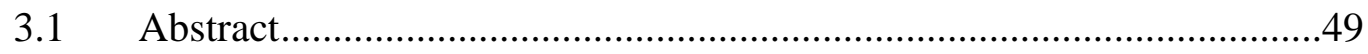

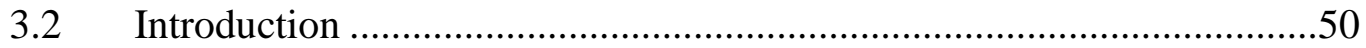

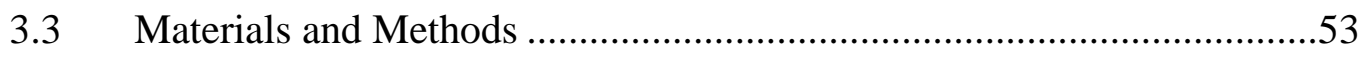

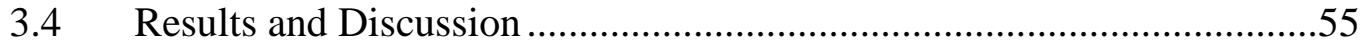

3.4.1 Palmer amaranth seed damage ..................................................55

3.4.2 Total Palmer amaranth seed germination ......................................56

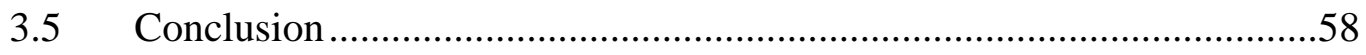

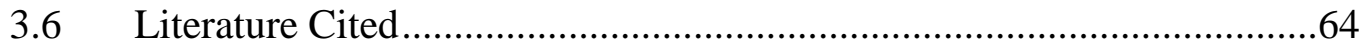

IV. EFFECT OF NITROGEN FERTILIZER APPLICATION RATE ON TARNISHED PLANT BUG (LYGUS LINEOLARIS PALISOT DE BEAUVOIS) INFESTATION IN PALMER AMARANTH (AMARANTHUS PALMERI S. WATS.) ............................................68

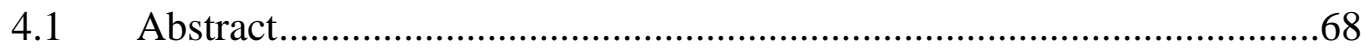

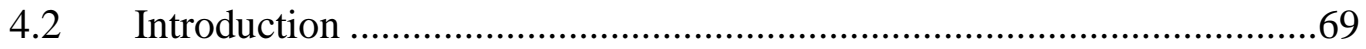

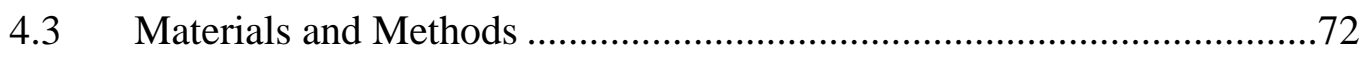

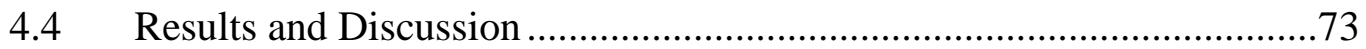

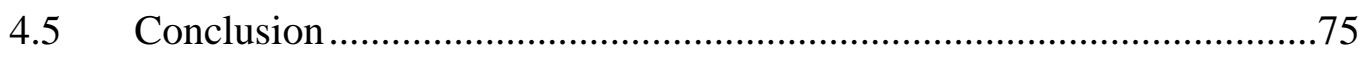

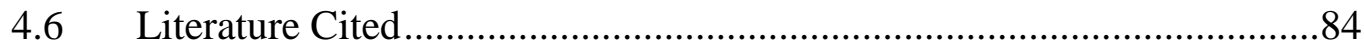

\section{APPENDIX}

A. VISUAL PALMER AMARANTH (AMARANTHUS PALMERI S. WATS.) CONTROL AS INFLUENCED BY INTERACTION DROPLET SIZE AND LOCATION INTERACTION .... 


\section{LIST OF TABLES}

Table 1.1 Year, location, GPS coordinates, Palmer amaranth density, application date, and weather conditions at the time of herbicide application.

Table 1.2 Herbicide, nozzle type, application pressure and droplet size classification for lactofen and acifluorfen application.

Table 1.3 Analysis of variance (ANOVA) probability values at each rating period for site-year, droplet size, and the interaction between droplet size and site-year with respect to visual Palmer amaranth control following lactofen and acifluorfen application.

Table 1.4 Analysis of variance (ANOVA) probability values for site-year, droplet size, and the interaction between droplet size and siteyear with respect to Palmer amaranth dry biomass following lactofen and acifluorfen application.

Table 1.5 Visual Palmer amaranth control following lactofen application with different spray droplet sizes.

Table 1.6 Visual Palmer amaranth control following acifluorfen application with various droplet sizes.

Table 1.7 Influence of droplet size on Palmer amaranth dry biomass at 28 DAT following lactofen and acifluorfen application.

Table 2.1 Year, location, GPS coordinates, Palmer amaranth density, application date, and weather conditions at the time of herbicide application.

Table 2.2 Herbicide, nozzle type, application pressure and droplet size classification for acifluorfen droplet size treatments.

Table 2.3 Generalized additive model (GAM) smooth parameters and deviance explained for visual Palmer amaranth control and dry biomass reduction. 
Table 2.4 Generalized additive model (GAM) analysis for maximum visual Palmer amaranth control and dry biomass pooled across all site-years.

Table 2.5 Generalized additive model (GAM) analysis for Palmer amaranth visual control and dry biomass for each site pooled over years.

Table 2.6 Droplet size prediction based on generalized additive model (GAM) analysis to reach and maintain $90 \%$ of maximum Palmer amaranth control in Dundee, MS, Beaver City, NE, and Robinsonville, MS pooled across years.

Table 2.7 Generalized additive model (GAM) analysis for visual Palmer amaranth control for each site-year.

Table 2.8 Droplet size prediction based on generalized additive model (GAM) analysis to reach and maintain $90 \%$ of maximum Palmer amaranth control in Dundee, MS, Beaver City, NE, and Robinsonville, MS for each site-year.

Table 3.1 Analysis of variance probability values for normal and damaged Palmer amaranth (AMAPA) seeds in 2016 and 2017.

Table 3.2 Air temperature and precipitation averages in Starkville, MS during experiment duration in 2016 and 2017.

Table 3.3 Analysis of variance probability values for total Palmer amaranth (AMAPA) seed germination in 2016 and 2017.

Table 3.4 Total Palmer amaranth (AMAPA) seed germination as a result of flooding period and seed burial depth pooled across soil texture and year.

Table 4.1 Nitrogen fertilizer application and sampling interval dates in Dundee, MS in 2016, 2017, and 2018.

Table 4.2 Analysis of variance probability values for cumulative tarnished plant bug population density across years in Dundee, MS.

Table 4.3 Tarnished plant bug population density as influenced by nitrogen fertilizer rate in Dundee, MS in 2016, 2017, and 2018. . .78

Table 4.4 Regression of log cumulative tarnished plant bug population density with week and nitrogen fertilizer rate as source of variation in 2016, 2017 and 2018. 
Table 4.5 Palmer amaranth (AMAPA) height, density, and sex probability values respective to nitrogen fertilizer application rate pooled across years.

Table 4.6 Palmer amaranth (AMAPA) height as influenced by nitrogen fertilizer application rate pooled across years. . .80

Table A.1 Visual Palmer amaranth control by site-year at 7 DAT following acifluorfen application. .88 


\section{LIST OF FIGURES}

Figure 2.1 Proportion of Palmer amaranth visual control (upper) and dry biomass (lower) 28 days after treatment predicted using generalized additive model (GAM) analysis. The gray shaded area represents the $95 \%$ confidence intervals.

Figure 2.2 Proportion of Palmer amaranth visual control (upper) and dry biomass (lower) 28 days after treatment predicted using generalized additive model (GAM) analysis for Dundee, MS across three site-years. The gray shaded area represents the $95 \%$ confidence intervals.

Figure 2.3 Proportion of Palmer amaranth visual control (upper) and dry biomass (lower) 28 days after treatment predicted using generalized additive model (GAM) analysis for Beaver City, NE across two site-years. The gray shaded area represents the $95 \%$ confidence intervals.

Figure 2.4 Proportion of Palmer amaranth visual control (upper) and dry biomass (lower) 28 days after treatment predicted using generalized additive model (GAM) analysis for Robinsonville, MS across two site-years. The gray shaded area represents the $95 \%$ confidence intervals.

Figure 3.1 Experimental area at the R. R. Foil Plant Research Center in Starkville, MS.

Figure 3.2 Visual aspects used for damaged (left) and normal (right) Palmer amaranth seed characterization.

Figure 3.3 Palmer amaranth (AMAPA) seed characterization based on soil texture in 2016 and 2017

Figure 3.4 Palmer amaranth (AMAPA) seed characterization in response to flooding period pooled across seed burial depth, soil texture, and year. 
Figure 4.1 Contour graph for log-cumulative tarnished plant bug population density as influenced by nitrogen fertilizer application rate and sampling week in 2016.

Figure 4.2 Contour graph for log-cumulative tarnished plant bug population density as influenced by nitrogen fertilizer application rate and sampling week in 2017.

Figure 4.3 Contour graph for log-cumulative tarnished plant bug population density as influenced by nitrogen fertilizer application rate and sampling week in 2018. 


\section{CHAPTER I}

\section{DROPLET SIZE IMPACT ON LACTOFEN AND ACIFLUORFEN EFFICACY FOR PALMER AMANRATH (AMARANTHUS PALMERI S. WATS.) CONTROL}

\subsection{Abstract \\ Concerns exist regarding development of Palmer amaranth (Amaranthus palmeri}

S. Wats.) populations resistant to multiple herbicide modes of action (MOA) across the southern and midwestern United States. Therefore, efficacious and cost-effective application methods are needed to maximize herbicide effectiveness for Palmer amaranth control. Experiments were conducted at three locations in Mississippi and Nebraska in 2016, 2017, and 2018 to evaluate the influence of droplet size on lactofen and acifluorfen efficacy on Palmer amaranth control and biomass reduction. Results demonstrate that Palmer amaranth control does not differ due to lactofen droplet size. Although lactofen applied with $300 \mu \mathrm{m}$ (medium) and $900 \mu \mathrm{m}$ (ultra coarse) spray droplets provided greater Palmer amaranth dry biomass reduction compared to $450 \mu \mathrm{m}$ (very coarse) and $750 \mu \mathrm{m}$ (ultra coarse) droplets, these differences were not present in visual estimations of Palmer amaranth control. Conversely, acifluorfen applied with $300 \mu \mathrm{m}$ (medium) droplets resulted in the greatest Palmer amaranth control. In addition, with exception of applications made with $600 \mu \mathrm{m}$ (extremely coarse) droplets, acifluorfen applied with all other droplet sizes reduced Palmer amaranth dry biomass. Overall, ultra coarse spray 
droplets are recommended for lactofen application and $300 \mu \mathrm{m}$ (medium) spray droplets should be used to maximize Palmer amaranth control with acifluorfen.

\subsection{Introduction}

Herbicide application is a crucial process in weed management to ensure optimum control while minimizing physical drift. Herbicide drift not only contributes to environmental pollution but also reduces herbicide efficacy (Knoche, 1994). Therefore, optimum spray performance is environmentally and economically important. Pimentel (2005) stated the increased use of pesticides in current crop protection practices has resulted in increased awareness and concern of the risks associated with off-target movement of pesticides. These concerns necessitate the need to re-evaluate application practices.

The application process can be divided in four successive stages: deposition (amount that hits the target), retention (amount remaining on the target after impaction), uptake (amount of herbicide taken into the plant), and translocation (amount of herbicide translocated inside the plant) (De Cock et al., 2017; Zabkiewicz, 2007). Deposition and drift of herbicide particles is highly affected by the droplet size spectra produced by agricultural nozzles (Creech et al., 2015). Pesticide droplet size has a large impact on spray drift and may be more important for mitigation of spray drift than environmental factors such as wind speed, temperature and humidity (Frost and Ware, 1970; Combellack, 1982; Bird et al., 1996). Spray solution droplet size is characterized by the volume median diameter $\left(D_{v 0.5}\right)$ of the droplet spectra, which corresponds to the median diameter where half of the spray volume consists of droplets smaller and the other half of spray droplets larger than the median (Meyer et al., 2015). 
Chemical companies and nozzle manufacturers generally recommend nozzles that produce smaller droplet sizes for application of non-systemic (contact) herbicides. Smaller droplet sizes result in greater target coverage, thus increasing control (Ennis et al., 1963; McKinlay et al., 1972; Lake, 1977; Knoche, 1994). Nevertheless, research has demonstrated that spray droplets smaller than $140 \mu \mathrm{m}$ (fine) are more likely to move offtarget (Burt and Smith, 1974). Finer spray droplets remain suspended longer in the atmosphere compared to larger droplets and are often displaced more so by lateral air movement than by the vertical force of gravity (Nuyttens et al., 2007).

The use of coarser spray droplets is often recommended to reduce herbicide particle drift. Coarser spray droplets carry greater kinetic energy which maximizes droplet impact on the leaf surface (Reichenberger et al., 2007). Although using coarser spray droplets may reduce particle drift, reduction of herbicide efficacy on target weed species has been documented (Wolf, 2002). Etheridge et al., (2001) observed reduced broadleaf signalgrass [Urochloa platyphylla (Munro ex. C. Wright) R. D. Webster] and common cocklebur (Xanthium strumarium L.) control with glufosinate and paraquat following application with increased droplet size. In contrast, Berger et al., (2014) reported no differences on Palmer amaranth (Amaranthus palmeri S. Wats.) control with lactofen applied using fine and coarse droplets.

The diverging results from aforementioned experiments may be due to differences in weed species and chemistries. Morphological factors such as leaf structure, presence or absence of leaf trichomes, and cuticle thickness can also impact herbicide effectiveness (Norsworthy et al., 2001). McKinlay et al., (1974) reported better leaf retention of paraquat following application with smaller droplet sizes on upright grass species 
compared to broadleaf species with horizontal leaf disposition. In addition to morphological factors, formulation, presence of adjuvants, and inert formulation components influence herbicide efficacy (Mellendorf et al., 2013).

Spray droplet size can be affected by herbicide formulation, even when applied under similar conditions. Differences in $D_{v 0.5}$ of two glyphosate formulations containing isopropylamine salt as active ingredient were reported by Mueller and Womac (1997), which suggests differences in $D_{v 0.5}$ may be also correlated to inert ingredients added to the formulated product. The effect of adjuvants, either included in tank mixes or in different herbicide formulations, can also affect herbicide efficacy by altering droplet size and surface tension (Ryerse et al., 2004; Holloway et al., 2000; Spanoghe et al., 2007). Grichar and Dotray (2011) reported greater control of smellmelon (Cucumis melo L.) when lactofen was applied with spray adjuvants compared to application without adjuvants.

Different physiochemical, morphological, and environmental factors contribute to variability in efficacy of contact herbicides using a particular spray droplet size range. Weed management strategies which include optimal spray droplet sizes maximize efficacy of contact herbicides (Butts et al., 2018). Pulse width modulation (PWM) sprayers can be used to optimize pesticide application as they provide a constant spray droplet size and pressure across a wide range of equipment driving speeds while variably managing flow (Butts et al., 2018). In these systems, flow is controlled by an electronically-powered solenoid valve placed upstream of the nozzle (Giles and Comino, 1989). Flow is altered by controlling the time proportion in which the solenoid valve remains open. The time relative to valve opening and closure is denominated as the duty 
cycle. Typically, solenoid valves are pulsed on a frequency of $10 \mathrm{~Hz}$ (10 pulses per second) (Butts et al., 2018). In comparison to other variable rate application systems, PWM sprayers allow flow rate changes without altering application pressure. Solenoid valves assist with pressure maintenance across spray boom sections minimizing the risk of product rate application errors (Anglung and Ayers, 2003; Luck et al., 2011).

Additionally, PWM sprayers provide greater flexibility to operators as a 10:1 turndown ratio in flow rate can be produced with no pressure or nozzle modifications resulting in a constant droplet size while flow rate is adjusted by the duty cycle in response to driving speed (Giles et al., 1996; GopalaPillai et al., 1999).

Sprayers equipped with variable rate control operated by application pressure have slow response time and reduced specific droplet size production (Giles and Comino 1989). Conversely, previous research has shown that PWM duty cycle has minimum to no effect on spray droplet size when using non-venturi nozzles (Butts et al., 2019; Giles et al., 1996). Furthermore, when operated at or above $40 \%$ duty cycle, PWM sprayers reduce little to no negative impact on spray pattern and coverage (Butts et al., 2019a; Mangus et al., 2017). Hence, PMW sprayers may be used to sustain and deliver a specific droplet size and spray pattern application.

The widespread occurrence of glyphosate and acetolactate synthase (ALS) resistant Palmer amaranth populations has led to an increased use of protoporphyrinogen oxidase (PPO) inhibiting herbicides, especially in soybean [Glycine max (L.) Merr.] and cotton (Gossypium hirsutum L.) systems (Salas et al., 2016). The first Palmer amaranth population resistant to PPO-inhibiting herbicides was reported in Arkansas in 2011 (Heap, 2019). Consequently, biotypes resistant to fomesafen and lactofen have been 
documented in Tennessee and Illinois, respectively (Heap, 2019). In addition, fields located in the northern Mississippi Alluvial Valley are likely to be infested with Palmer amaranth biotypes resistant to PPO-inhibiting herbicides (Bond et al., 2016). Therefore, cost effective weed management practices that maximize herbicide efficacy and mitigate spray drift are needed to combat PPO-resistant Palmer amaranth and minimize further selection pressure for resistant biotypes. The objective of this experiment was to evaluate the influence of spray droplet size on lactofen and acifluorfen effectiveness for Palmer amaranth control.

\subsection{Materials and Methods}

Experiments were conducted in 2016, 2017, and 2018 in a non-crop environment in Dundee, MS (2016-2018) on a Sharkey clay soil, Beaver City, NE (2016-2017) on a Holdrege silt loam, and Robinsonville, MS (2017-2018) on a Commerce silt loam to evaluate the effect of spray droplet size using lactofen and acifluorfen for Palmer amaranth control (Table 1.1). Lactofen (Cobra ${ }^{\circledR}$, Valent U.S.A. Corporation, Walnut Creek, CA 94596) at $0.21 \mathrm{~kg}$ ai ha ${ }^{-1}$ and acifluorfen (Ultra Blazer ${ }^{\circledR}$, UPL Corporate, King of Prussia, PA 19406) at $0.42 \mathrm{~kg}$ ai ha ${ }^{-1}$ were applied with crop oil concentrate (AgriDex ${ }^{\circledR}$, Helena Chemical Co., Collierville, TN 38017) at $1 \%$ v/v to $15 \mathrm{~cm}$ tall Palmer amaranth plants. Treatments consisted of six targeted droplet sizes (150, 300, 450, 600, 750 , and $900 \mu \mathrm{m})$ determined from the volume median diameter $\left(D_{v 0.5}\right)$ of the measured droplet size distribution. Herbicides were evaluated as two different experiments. One nontreated control per site-year in each experiment was used for treatment comparison. Plot dimension was 4 meters wide by 12 meters long. Treatments were arranged in a randomized complete block design with four replications. Treatments were applied using 
a tractor mounted sprayer equipped with a Pin Point ${ }^{\circledR}$ pulse width modulation (PWM) sprayer (Capstan Ag Systems, Inc., Topeka, KA 66609) and with non-venturi Wilger ${ }^{\mathrm{TM}}$ precision spray tips (Wilger Inc., Lexington, TN 38351) operated at $4.8 \mathrm{~km} \mathrm{~h}^{-1}$ and spray volume of $140 \mathrm{~L} \mathrm{ha}^{-1}$.

Prior to experiment initiation, droplet size spectra for lactofen and acifluorfen was characterized in a low-speed wind tunnel located at the Pesticide Application Technology (PAT) Laboratory in North Platte, Nebraska. Nozzle type, orifice size, and application pressure necessary to produce the aforementioned droplet sizes for each herbicide solution were determined using a Sympatec HELOS-VARIO/KR laser diffraction system (Sympatec Inc., Clausthal-Zellerfeld, Germany 38678) equipped with R7 lens capable of detecting particle sizes ranging from 18 to $3500 \mu \mathrm{m}$ (Table 1.2). Procedures for actual droplet size determination follow those described by Butts et al., (2018). Droplet size classifications were assigned in accordance with ASABE S572.1 (ASABE, 2009). Visual Palmer amaranth control evaluations were collected at 7, 14, 21, and 28 days after herbicide treatment (DAT). Palmer amaranth control was evaluated on a scale of 0 (no control) to $100 \%$ (complete death of all plants) relative to the nontreated check (Frans et al., 1986). Prior to herbicide application, 10 plants per plot were tagged at soil surface for above ground dry biomass evaluation. At the end of the experiment, tagged plants were harvested, placed in paper bags, removed from experimental area, and dried in a forced air dryer at $55^{\circ} \mathrm{C}$ for 72 hours. Tagged plants also serve as a reference to weed control evaluation in plots where new emergence and/or regrowth occurred. Visual Palmer amaranth control and dry aboveground biomass data were subjected to analysis of variance (ANOVA) using PROC MIXED procedure in SAS v.9.4 (SAS® Institute Inc., 
Cary, NC 27513). Treatment means were separated using Fisher's Protected LSD at 0.05 level of significance. Fixed factors were spray droplet size, year, and location. Given the differences in the number of years in which experiments were conducted at each location, year and location were combined in one factor (site-year). The absence of an interaction between spray droplet size and location along with similar result trend were used as a parameter for data pooling over site-years. The nontreated was not included in visual Palmer amaranth control analysis to allow greater mean separation between response parameters. Visual Palmer amaranth control and dry biomass data were analyzed by rating period $(7,14,21$, and $28 \mathrm{DAT})$ to better evaluate responses following herbicide application.

\section{$1.4 \quad$ Results and Discussion}

No droplet size by site-year interaction was present for visual Palmer amaranth control at any rating interval for either herbicide, except acifluorfen at 7 DAT (Table 1.3). Visual Palmer amaranth control data analysis at 7 DAT within each site-year is

presented in Appendix A. At 14, 21, and 28 DAT the interaction between droplet size and site-year was not significant. Furthermore, the same trend of results was observed across all site-years. Therefore, visual Palmer amaranth control data were pooled across all siteyears (Table 1.3). No interaction between droplet size and site-year was present for Palmer amaranth dry biomass 28 days after lactofen and acifluorfen application. A similar trend in dry biomass reduction was present across site-years, hence dry biomass data were pooled over site-years (Table 1.4). 


\subsubsection{Palmer amaranth control with lactofen}

Droplet size did not affect lactofen efficacy on Palmer amaranth, regardless of rating period (Table 1.3). Visual Palmer amaranth control at 7 DAT following lactofen application ranged from 68 to $77 \%$ (Table 1.5). At 14, 21, and 28 DAT visual Palmer amaranth control ranged from 63 to $69 \%, 61$ to $66 \%$, and 56 to $62 \%$, respectively (Table 1.5). These results are consistent with previous research conducted by Berger et al., (2014) who reported no differences in Palmer amaranth control with lactofen using XR flat fan and air induction (AI) nozzles. Similarly, Sikkema et al., (2008) reported no differences in common lambsquarters (Chenopodium album L.) control using fomesafen with, flat fan or air induction nozzles. Palmer amaranth control below $80 \%$ observed in the presented studies is a result of herbicide application to taller plants. Plants were allowed to grow to $15 \mathrm{~cm}$ in order to separate control due to droplet size. Previous research has demonstrated increased weed control when lactofen and acifluorfen were applied to plants smaller than $10 \mathrm{~cm}$ in height (Grichar, 2007; Hager et al., 2003).

Lactofen application reduced Palmer amaranth dry biomass compared to the nontreated (Table 1.7). Lactofen applied with $300 \mu \mathrm{m}$ (medium) and $900 \mu \mathrm{m}$ (ultra coarse) droplets provided the greatest Palmer amaranth dry biomass reduction (Table 1.7). However, lactofen applied with $600 \mu \mathrm{m}$ (extremely coarse) and $150 \mu \mathrm{m}$ (fine) droplets provided similar dry biomass reduction. The abundant genetic variability and morphology within Palmer amaranth populations could be responsible for the increased variability in dry biomass. Bravo et al., (2017) reported significant differences in morphology and growth traits among ten Palmer amaranth populations from Florida and Georgia. 


\subsubsection{Palmer amaranth control with acifluorfen}

Acifluorfen applied with $300 \mu \mathrm{m}$ (medium) droplets provided the greatest visual Palmer amaranth control at 14, 21, and 28 DAT (Table 1.6). Overall, Palmer amaranth control following acifluorfen application with $300 \mu \mathrm{m}$ (medium) spray droplets was 10, 13, and $13 \%$ greater compared to all other droplet sizes at 14, 21, and 28 DAT, respectively (Table 1.6). De Cock et al., (2017) reported that herbicides applied with 250 $\mu \mathrm{m}$ (medium) spray droplets had increased leaf deposition. Furthermore, herbicide application with spray droplets ranging between $200 \mu \mathrm{m}$ (fine) and $270 \mu \mathrm{m}$ (medium) have lower spray drift potential and reduced leaf shattering and bouncing (De Cock et al., 2017). Previous research conducted by Spillman (1984) reported reduced Palmer amaranth control when acifluorfen was applied with $150 \mu \mathrm{m}$ (fine) and $450 \mu \mathrm{m}$ (very coarse) spray droplets which may be a result of increased particle drift and droplet shattering, respectively. Additionally, Shaw et al., (2000) observed increased common cocklebur (Xathium strumarium L.) control following acifluorfen application with 350 $\mu \mathrm{m}$ (coarse) spray droplets.

Differences in Palmer amaranth dry biomass did not correspond to those observed in visual Palmer amaranth control (Table 1.7). Acifluorfen applied with all droplet sizes except $600 \mu \mathrm{m}$ (extremely coarse) reduced Palmer amaranth dry biomass compared to the nontreated (Table 1.7). Altering size, biomass, resource allocation and phenology are manners by which Palmer amaranth normally responds to stress conditions such as herbicide application (Korres and Norsworthy, 2017). These characteristics may contribute to differences observed between visual weed control and dry biomass. 


\subsection{Conclusion}

Spray droplet size did not influence lactofen efficacy on Palmer amaranth, regardless of rating period. Spray droplet sizes ranging from $150 \mu \mathrm{m}$ (fine) to $900 \mu \mathrm{m}$ (ultra coarse) optimized lactofen efficacy. However, in order to mitigate spray drift preference should be given to coarser droplet sizes. Additionally, acifluorfen applied with $300 \mu \mathrm{m}$ (medium) spray droplets resulted in the greatest level of Palmer amaranth control. Acifluorfen should be applied using $300 \mu \mathrm{m}$ (medium) spray droplets to maximize Palmer amaranth control. These experiments also highlight the importance of PWM sprayers in increasing application precision and flexibility to increase herbicide efficacy in different environments. The use of PWM sprayers along with proper nozzle type and pressure combinations could effectively maximize lactofen and acifluorfen efficacy for Palmer amaranth control and improve spray drift mitigation efforts. 
Table 1.1 Year, location, GPS coordinates, Palmer amaranth density, application date, and weather conditions at the time of herbicide application.

\begin{tabular}{|c|c|c|c|c|c|c|c|}
\hline & \multirow[b]{2}{*}{ Location } & \multirow[b]{2}{*}{ GPS Coordinates } & \multirow[b]{2}{*}{$\begin{array}{l}\text { AMAPA } \\
\text { Density }^{\text {a }}\end{array}$} & \multirow[b]{2}{*}{ Date } & \multicolumn{3}{|c|}{ Weather conditions at application } \\
\hline Year & & & & & Wind speed & $\begin{array}{c}\text { Air } \\
\text { temperature }\end{array}$ & $\begin{array}{l}\text { Relative } \\
\text { humidity }\end{array}$ \\
\hline & \multirow{3}{*}{ Dundee, MS } & & Plants $\mathrm{m}^{-2}$ & & $\mathrm{~km} \mathrm{~h}^{-1}$ & ${ }^{\circ} \mathrm{C}$ & $\%$ \\
\hline \multirow[t]{2}{*}{2016} & & $34^{\circ} 32^{\prime} 39^{\prime \prime} \mathrm{N}$ & 140 & 08 Sept. & 5 & 21 & 40 \\
\hline & & $90^{\circ} 28^{\prime} 22^{\prime \prime} \mathrm{W}$ & & & & & \\
\hline \multirow[t]{2}{*}{2016} & \multirow[t]{2}{*}{ Beaver City, NE } & $40^{\circ} 14^{\prime} 2 ” \mathrm{~N}$ & 100 & 07 June & 6 & 22 & 40 \\
\hline & & $98^{\circ} 57^{\prime} 10^{\prime \prime} \mathrm{W}$ & & & & & \\
\hline \multirow[t]{2}{*}{2017} & \multirow[t]{2}{*}{ Dundee, MS } & $34^{\circ} 32^{\prime} 39^{\prime \prime} \mathrm{N}$ & 334 & 01 June & 14 & 25 & 74 \\
\hline & & $90^{\circ} 28^{\prime} 22^{\prime \prime} \mathrm{W}$ & & & & & \\
\hline \multirow[t]{2}{*}{2017} & \multirow[t]{2}{*}{ Beaver City, NE } & $40^{\circ} 14^{\prime} 2 ” \mathrm{~N}$ & 25 & 22 Aug. & 10 & 23 & 55 \\
\hline & & $98^{\circ} 57^{\prime} 10^{\prime \prime} \mathrm{W}$ & & & & & \\
\hline \multirow[t]{2}{*}{2017} & \multirow[t]{2}{*}{ Robinsonville, MS } & $34^{\circ} 49^{\prime} 41^{\prime \prime} \mathrm{N}$ & 288 & 01 June & 5 & 29 & 60 \\
\hline & & $90^{\circ} 17^{\prime} 21^{\prime \prime} \mathrm{W}$ & & & & & \\
\hline \multirow[t]{2}{*}{2018} & \multirow[t]{2}{*}{ Dundee, MS } & $34^{\circ} 32^{\prime} 39^{\prime \prime} \mathrm{N}$ & 217 & 25 June & 16 & 32 & 82 \\
\hline & & $90^{\circ} 28^{\prime} 22^{\prime \prime} \mathrm{W}$ & & & & & \\
\hline \multirow[t]{2}{*}{2018} & \multirow[t]{2}{*}{ Robinsonville, MS } & $34^{\circ} 49^{\prime} 41^{\prime \prime} \mathrm{N}$ & 200 & 15 June & 2 & 33 & 51 \\
\hline & & $90^{\circ} 17^{\prime} 21^{\prime \prime} \mathrm{W}$ & & & & & \\
\hline
\end{tabular}

${ }^{\text {a }}$ Palmer amaranth (AMAPA) population density (plants $\mathrm{m}^{-2}$ ) collected one week prior herbicide application date. 
Table 1.2 Herbicide, nozzle type, application pressure and droplet size classification for lactofen and acifluorfen application.

\begin{tabular}{lcccccc}
\hline \multirow{3}{*}{ Herbicide } & Nozzle & $\begin{array}{c}\text { Application } \\
\text { pressure }\end{array}$ & $\begin{array}{c}\text { Target } \\
\text { droplet } \\
\text { size }\end{array}$ & $\begin{array}{c}\text { Actual } \\
\text { droplet } \\
\text { size }^{\mathrm{b}}\end{array}$ & $\begin{array}{c}\text { Standard } \\
\text { error }\end{array}$ & $\begin{array}{c}\text { Spray } \\
\text { classification }^{\mathrm{c}}\end{array}$ \\
\hline \multirow{5}{*}{ Lactofen } & ER 110015 & 483 & 150 & 169 & 0.89 & $\mathrm{~F}$ \\
& SR 11004 & 379 & 300 & 297 & 0.68 & $\mathrm{M}$ \\
& MR 11006 & 207 & 450 & 452 & 0.77 & VC \\
& DR 11005 & 248 & 600 & 600 & 2.04 & EC \\
& UR11008 & 379 & 750 & 744 & 1.09 & UC \\
& UR 11010 & 241 & 900 & 903 & 1.97 & UC \\
& ER 110015 & 414 & 150 & 153 & 0.60 & F \\
& SR 11004 & 324 & 300 & 300 & 3.58 & M \\
& DR 11003 & 414 & 450 & 453 & 0.98 & VC \\
& DR11006 & 331 & 600 & 597 & 0.73 & EC \\
& UR11006 & 345 & 750 & 746 & 1.95 & UC \\
& UR 11010 & 276 & 900 & 904 & 3.46 & UC \\
\hline
\end{tabular}

${ }^{\mathrm{a}}$ Flat fan, non-venturi nozzles; Wilger ${ }^{\mathrm{TM}}$ precision spray tips (Wilger Inc., Lexington, TN 38351).

${ }^{\mathrm{b}}$ Actual droplet size was measured using nozzle and application pressure combinations for each herbicide.

${ }^{\mathrm{c}}$ Spray classification according to ASABE S572.1 where F=Fine, M=Medium, VC=Very Coarse, EC=Extremely Coarse, and UC=Ultra Coarse. 
Table 1.3 Analysis of variance (ANOVA) probability values at each rating period for site-year, droplet size, and the interaction between droplet size and siteyear with respect to visual Palmer amaranth control following lactofen and acifluorfen application.

\begin{tabular}{ccccc}
\hline Herbicide & Rating period & Site-year & Droplet size & $\begin{array}{c}\text { Site-year* } \\
\text { droplet size }\end{array}$ \\
\hline \multirow{2}{*}{ Lactofen } & & $------------------p-$-values & a------------------- \\
& 7 & $<0.0001$ & 0.2257 & 0.6572 \\
& 14 & $<0.0001$ & 0.6140 & 0.8333 \\
Acifluorfen & 21 & $<0.0001$ & 0.7929 & 0.7954 \\
& 28 & $<0.0001$ & 0.7946 & 0.8812 \\
& 14 & $<0.0001$ & 0.0004 & 0.0004 \\
& 21 & $<0.0001$ & 0.0086 & 0.0709 \\
& 28 & $<0.0001$ & 0.0015 & 0.7317 \\
\hline
\end{tabular}

${ }^{a}$ Probability values calculated based on data pooled across seven site-years.

Table 1.4 Analysis of variance (ANOVA) probability values for site-year, droplet size, and the interaction between droplet size and site-year with respect to Palmer amaranth dry biomass following lactofen and acifluorfen application.

\begin{tabular}{cccc}
\hline Herbicide & Site-year & Droplet size & $\begin{array}{c}\text { Site-year*droplet } \\
\text { size }\end{array}$ \\
\hline Lactofen & $------------------------------p-$ values $^{\mathrm{a}}$---------------------------- \\
Acifluorfen & $<0.0001$ & $<0.0001$ & 0.0865 \\
& $<0.0001$ & 0.0359 & 0.7991 \\
\hline
\end{tabular}

${ }^{a}$ Probability values calculated based on data pooled across seven site-years. 
Table 1.5 Visual Palmer amaranth control following lactofen application with different spray droplet sizes.

\begin{tabular}{lcccc}
\hline & \multicolumn{4}{c}{ Days after treatment (DAT) ${ }^{\mathrm{a}}$} \\
\hline Droplet size & 7 & 14 & 21 & 28 \\
\hline$\mu \mathrm{m}$ & 71 & 65 & 62 & 59 \\
150 & 69 & 63 & 61 & 56 \\
300 & 69 & 68 & 66 & 62 \\
450 & 77 & 69 & 66 & 62 \\
600 & 68 & 64 & 61 & 60 \\
750 & 70 & 63 & 63 & 59 \\
900 & $\mathrm{NS}$ & $\mathrm{NS}$ & $\mathrm{NS}$ & $\mathrm{NS}$ \\
LSD $(0.05)^{\mathrm{b}}$ & & & & \\
\hline
\end{tabular}

a Visual Palmer amaranth control data were pooled across seven site-years and analyzed within each rating period.

${ }^{\mathrm{b}}$ Visual Palmer amaranth control did not differ at any rating period following lactofen application. 
Table 1.6 Visual Palmer amaranth control following acifluorfen application with various droplet sizes.

\begin{tabular}{lccc}
\hline & \multicolumn{3}{c}{ Days after treatment (DAT) ${ }^{\mathrm{a}, \mathrm{b}}$} \\
\hline Droplet size & 14 & 21 & 28 \\
\hline$\mu \mathrm{m}$ & $53 \mathrm{~b}$ & $53 \mathrm{~b}$ & $56 \mathrm{bc}$ \\
150 & $62 \mathrm{a}$ & $63 \mathrm{a}$ & $67 \mathrm{a}$ \\
300 & $42 \mathrm{c}$ & $46 \mathrm{~b}$ & $52 \mathrm{c}$ \\
450 & $52 \mathrm{~b}$ & $51 \mathrm{~b}$ & $56 \mathrm{bc}$ \\
600 & $47 \mathrm{bc}$ & $46 \mathrm{~b}$ & $59 \mathrm{abc}$ \\
750 & $49 \mathrm{bc}$ & $54 \mathrm{~b}$ & $60 \mathrm{ab}$ \\
900 & & ------ & $\%$ \\
\hline
\end{tabular}

${ }^{a}$ Visual Palmer amaranth control data were pooled across seven site-years and analyzed within each rating period.

${ }^{\mathrm{b}}$ Means within a column followed by the same letter are not significantly different according to Fisher's protected LSD $(\alpha=0.05)$. 
Table 1.7 Influence of droplet size on Palmer amaranth dry biomass at 28 DAT following lactofen and acifluorfen application.

\begin{tabular}{lcc}
\hline & \multicolumn{2}{c}{ Herbicide $^{\mathrm{a}, \mathrm{b}}$} \\
\hline Droplet size & Lactofen & Acifluorfen \\
\hline$\mu \mathrm{m}$ & $121 \mathrm{a}$ & $168 \mathrm{a}$ \\
Nontreated & $69 \mathrm{bc}$ & $102 \mathrm{~b}$ \\
150 & $54 \mathrm{c}$ & $106 \mathrm{~b}$ \\
300 & $89 \mathrm{~b}$ & $123 \mathrm{~b}$ \\
450 & $75 \mathrm{bc}$ & $134 \mathrm{ab}$ \\
600 & $91 \mathrm{~b}$ & $109 \mathrm{~b}$ \\
750 & $57 \mathrm{c}$ & $123 \mathrm{~b}$ \\
900 & & prams/10 plants \\
\hline
\end{tabular}

${ }^{a}$ Palmer amaranth dry biomass data were pooled across seven site-years.

${ }^{\mathrm{b}}$ Means within a column followed by the same letter are not significantly different according to Fisher's protected LSD $(\alpha=0.05)$. 


\subsection{Literature Cited}

[ASABE] American Society of Agricultural and Biological Engineers. 2009. Spray nozzle classification by droplet spectra. St. Joseph, MI: ASABE Standard S572.1. pp. 1-3.

Anglund, E.A., Ayers, P.D. 2003. Field evaluation of response times for a variable rate (pressure-based and injection) liquid chemical applicator. Appl Eng Agric 19:273-282.

Berger, S.T., Dobrow, M.H., Ferrell, J.A., Webster, T.M. 2014. Influence of carrier volume and nozzle selection on palmer amaranth control. Peanut Sci 41(2):120123.

Bird, S.L., Esterly, D.M., Perry, S.G. 1996. Atmospheric pollutants and trace gases. Offtarget deposition of pesticides from agricultural aerial spray applications. J Environ Qual 25:1095-1104.

Bond, J.A., Reynolds, D.B., Irby, T. 2016. Managing PPO-resistant Palmer amaranth in Mississippi soybean. Mississippi State University Ext. www.mississippicrops.com/2016/03/25/managing-ppo-resistant-palmer-amaranth-in-mississippisoybean/ (accessed 14 Jan. 2019)

Bravo, W., Leon, R.G., Ferrell, J.A., Mulvaney, M.J., Wood, C.W. 2018. Evolutionary adaptations of Palmer amaranth (Amaranthus palmeri) to nitrogen fertilization and crop rotation history affect morphology and nutrient-use efficiency. Weed Sci 66(2):180-189.

Burt, E.C., Smith, D.B. 1974. Effects of droplet sizes on deposition of ULV spray. J Econ Entomol 67:751-754.

Butts, T.R., Samples, C.A., Franca, L.X., Dodds, D.M., Reynolds, D.B., Adams, J.W., Zollinger, R.K., Howatt, K.A., Fritz, B.K., Clint Hoffmann, W., Kruger, G.R. 2018. Spray droplet size and carrier volume effect on dicamba and glufosinate efficacy. Pest Manag Sci 74(9):2020-2029.

Butts, T.R., Butts, L.E., Luck, J.D., Fritz, B.K., Hoffmann, W.C., Kruger, G.R. 2019. Droplet size and nozzle tip pressure from a pulse-width modulation sprayer. J Biosyst Eng 178:52-69.

Butts, T.R., Luck, J.D., Fritz, B.K., Hoffmann, W.C., Kruger, G.R. 2019a. Evaluation of spray pattern uniformity using three unique analyses as impacted by nozzle, pressure, and pulse-width modulation duty cycle. Pest Manag Sci., DOI: $10.1002 /$ ps.5352. 
Combellack, J.H. 1982. Loss of herbicides from ground sprayers. Weed Res 22:193-204.

Creech, C.F., Henry, R.S., Fritz, B.K., Kruger, G.R. 2015. Influence of herbicide active ingredient, nozzle type, orifice size, spray pressure, and carrier volume rate on spray droplet size characteristics. Weed Technol 29:298-310.

De Cock, N., Massinon, M., Salah, S. O., Lebeau, F. 2017. Investigation on optimal spray properties for ground based agricultural applications using deposition and retention models. J. Biosyst Eng 162:99-111.

Ennis, W.B., Williamson. R.E. 1963. Influence of droplet size on effectiveness of lowvolume herbicidal sprays. Weeds 11:67-72.

Etheridge, R.E., Hart, W.E., Hayes, R.M., Mueller, T.C. 2001. Effect of venturi-type nozzles and application volume on postemergence herbicide efficacy. Weed Technol 15:75-80.

Frans, R.E., Talbert, R., Marx, D., Crowley, H. 1986. Experimental design and techniques for measuring and analyzing plant responses to weed control practices. pp. 37-38. in N.D. Camper (ed.) Research Methods in Weed Science. 3rd ed. Champaign, IL: Southern Weed Science Society.

Frost, K.R., Ware, G.W. 1970. Pesticide drift from aerial and ground applications. Agric Eng 51(8):460-464.

Giles, D.K., Comino, J.A. 1989. Variable flow control for pressure atomization nozzles. J Commerical Veh SAE Trans 98:237-249.

Giles, D.K., Henderson, G.W., Funk, K. 1996. Digital control of flow rate and spray droplet size from agricultural nozzles for precision chemical application. Precis Agric 729-738.

GopalaPillai, S., Tian, L., Zheng, J. 1999. Evaluation of a flow control system for sitespecific herbicide applications. Trans ASABE 42:863-870.

Grichar, W.J., Dotray, P.A. 2011. Controlling weeds found in peanut with lactofen. Crop Manag 1:0-0.

Hager, A.G., Wax, L.M., Bollero, G.A., Stoller, E.W. 2003. Influence of diphenylether herbicide application rate and timing on common waterhemp (Amaranthus rudis) control in soybean (Glycine max). Weed Technol 17(1):14-20.

Heap, I.M. 2019. The International survey of herbicide-resistant weeds. Available at: www.weedscience.org. Accessed 12 January, 2019. 
Holloway, P.J., Butler Ellis, M.C., Webb, D.A., Western, N.M., Tuck, C.R., Hayes, A.L., Miller, P.C.H. 2000. Effects of some agricultural tank-mix adjuvants on the deposition efficiency of aqueous sprays on foliage. Crop Prot 19:27-37.

Knoche, M. 1994. Effect of droplet size and carrier volume on performance of foliageapplied herbicides. Crop Prot 13:163-178.

Korres, N.E., Norsworthy, J.K., Burgos, N.R., Oosterhuis, D.M. 2017. Temperature and drought impacts on rice production: An agronomic perspective regarding shortand long-term adaptation measures. Water resources and rural development 9:1227.

Lake, J.R. 1977. The effect of drop size and velocity on the performance of agricultural sprays. Pestic Sci 8:515-520.

Luck, J.D., Sharda, A., Pitla, S.K., Fulton, J.P., Shearer, S.A. 2011. A case study concerning the effects of controller response and turning movements on application rate uniformity with a self-propelled sprayer. Trans ASABE 54:423431.

Mangus, D.L., Sharda, A., Engelhardt, A., Flippo, D., Strasser, R., Luck, J.D., Griffin, T. 2017. Analyzing the nozzle spray fan pattern of an agricultural sprayer using pulse-width modulation technology to generate an on-ground coverage map. Trans ASABE 60:315-325.

McKinlay, K.S., Brandt, S.A., Morse, P., Ashford, R. 1972. Droplet size and phytotoxicity of herbicides. Weed Sci 20:450-452.

McKinlay, K.S., Ashford, R., Ford, R.J. 1974. Effects of drop size, spray volume, and dosage on paraquat toxicity. Weed Sci 22:31-34

Mellendorf, T.G., Young, J.M., Matthews, J.L., Young, B.G. 2013. Influence of plant height and glyphosate on saflufenacil efficacy on glyphosate-resistant horseweed (Conyza canadensis). Weed Technol 27(3):463-467.

Meyer, C. J., Norsworthy, J. K., Kruger, G. R., Barber, T. 2015. Influence of droplet size on efficacy of the formulated products Engenia ${ }^{\mathrm{TM}}$, Roundup PowerMax ${ }^{\circledR}$, and Liberty®. Weed Technol 29(4):641-652.

Mueller, T.C., Womac, A.R. 1997. Effect of formulation and nozzle type on droplet size with isopropylamine and trimesium salts of glyphosate. Weed Technol 11:639643.

Norsworthy, J.K., Burgos, N.R., Oliver, L.R. 2001. Differences in weed tolerance to glyphosate involve different mechanisms. Weed Technol 15:725-731. 
Nuyttens, D., Baetens, K., De Schampheleire, M., Sonck, B. 2007. Effect of nozzle type, size and pressure on spray droplet characteristics. Biosyst Eng 97:333-345.

Pimentel, D. 2005. Environmental and economic costs of the application of pesticides primarily in the United States. Environ Dev Sustain 7(2):229-252.

Reichenberger, S., Bach, M., Skitschak, A., Frede, H.G. 2007. Mitigation strategies to reduce pesticide inputs into ground and surface water and their effectiveness; a review. Sci Total Environ 384:1-35.

Ryerse, J.S., Downer, R.A., Sammons, D., Feng, P.C.C. 2004. Effect of glyphosate spray droplets on leaf cytology in velvetleaf (Abutilon theophrasti). Weed Sci 52:302309.

Salas, R.A., Burgos, N.R., Tranel, P., Singh, S., Glasglow, L., Scott, R.C., Nichols, R.I. 2016. Resistance to PPO-inhibiting herbicide in Palmer amaranth from Arkansas. Pest Manag Sci 72(5):864-869.

Shaw, D.R., Morris, W.H., Webster, E.P., Smith, D.B. 2000. Effects of spray volume and droplet size on herbicide deposition and common cocklebur (Xanthium strumarium) control. Weed Technol 14(2):321-326.

Sikkema, P.H., Brown, L., Shropshire, C., Spieser, H., Soltani, N. 2008. Flat fan and air induction nozzles affect soybean herbicide efficacy. Weed Biol Manag 8(1):3138.

Spanoghe, P., De Schampheleire, M., Van, D.M., Steurbaut, W. 2007. Influence of agricultural adjuvants on droplet spectra. Pest Manag Sci 63:4-16.

Spillman, J.J. 1984. Spray impaction, retention and adhesion: An introduction to basic characteristics. Pest Manag Sci 15:97-106.

Wolf, T.M. 2002. Optimizing herbicide performance - biological consequences of using low-drift nozzles. Asp Appl Biol 66:79-86.

Zabkiewicz, J.A. 2007. Spray formulation efficacy-holistic and futuristic perspectives. Crop Prot 26:312-319. 


\section{CHAPTER II}

\section{PALMER AMARANTH (AMARANTHUS PALMERI S. WATS.) CONTROL USING VARIOUS DROPLET SIZES OF ACIFLUORFEN}

\subsection{Abstract}

The widespread occurrence of glyphosate and acetolactate synthase (ALS) resistant Palmer amaranth (Amaranthus palmeri S. Wats.) populations has led to increased use of protoporphyrinogen oxidase (PPO) inhibiting herbicides. Acifluorfen is a non-systemic PPO-inhibiting herbicide commonly used for postemergence Palmer amaranth control in soybean [Glycine max (L.) Merr.], peanut (Arachis hypogaea L.), and rice (Oryza sativa L.) across the southern United States. In recent years, concerns have been raised regarding herbicide selection pressure and particle drift, increasing the need for application practices that optimize herbicide efficacy while mitigating spray drift. Experiments were conducted at three locations in Mississippi and Nebraska in 2016, 2017, and 2018 for a total of seven site-years to evaluate the influence of spray droplet size $[150 \mu \mathrm{m}$ (fine) to $900 \mu \mathrm{m}$ (ultra coarse)] on acifluorfen effectiveness for Palmer amaranth control. Generalized additive model (GAM) analysis suggests that acifluorfen applied with $150 \mu \mathrm{m}$ (fine) droplets provides the greatest visual Palmer amaranth control and biomass reduction when data were pooled across all site-years. To maintain satisfactory visual Palmer amaranth control (greater or equal to $90 \%$ of maximum observed control) and mitigate drift potential, droplet sizes up to $425 \mu \mathrm{m}$ (very coarse) 
may be utilized. However, prediction models were substantially stronger when visual control observations were made at individual testing location. Prediction models indicated that 250 (medium), 150 (fine), and $370 \mu \mathrm{m}$ (coarse) droplets maximized Palmer amaranth control in Dundee, MS, Beaver City, NE, and Robinsonville, MS, respectively. Droplet sizes between 180 (fine) to $310 \mu \mathrm{m}$ (medium) and 150 (fine) to $340 \mu \mathrm{m}$ (medium) are recommended to sustain at least 90\% Palmer amaranth control in Dundee, MS and Beaver City, NE, respectively. Furthermore, spray droplets between 220 (medium) to $680 \mu \mathrm{m}$ (ultra coarse) are recommended to maintain at least 90\% Palmer amaranth control in Robinsonville, MS. The influence of droplet size on acifluorfen efficacy for Palmer amaranth control is location-specific. Prediction models should be developed for individual locations to maximize herbicide efficacy and optimize drift mitigation efforts.

\section{$2.2 \quad$ Introduction}

The objective of postemergence herbicide application is to deliver the proper amount of spray solution to the leaf surface of targeted plant species (Ennis and Williamson, 1963). Ideally, the amount of solution applied by the sprayer should provide uniform spray deposition across the target maximizing the amount of herbicide available for uptake (Shaw et al., 2000). Optimizing chemical deposition through the use of proper droplet size could maximize herbicide efficacy, thus increasing weed control. Previous research has demonstrated that spray application is effective, but in several cases inefficient (Knoche, 1994; Beyer, 1991). Normally, a small fraction of active ingredient being applied is responsible for the desired plant response. Most of the spray solution does not hit the target and potentially contributes to particle drift (Knoche, 1994). 
Therefore, greater application precision and efficiency is economically and ecologically beneficial.

Several factors affect deposition and retention of pesticide spray droplets. Meteorological factors such as wind speed, air temperature and humidity, and atmospheric stability; application factors such as sprayer, nozzle type, nozzle size, application pressure, spray boom height, angle, and driving speed; and chemical formulation can all influence the effectiveness of a given compound (Carlsen et al., 2006). Research has demonstrated that among these factors, droplet size is critical to spray deposition and drift (Taylor et al., 2004; Whisenant et al., 1993; Yates et al., 1976).

The droplet spectra of a pesticide is composed of spray droplets with various sizes and is characterized by the volume median diameter $\left(D_{v 50}\right)$ of the spray solution, where half of the spray droplets are smaller and half larger than the median (Meyer et al., 2015). Typically, spray droplets are classified by their diameter presented in micrometers $(\mu \mathrm{m})$. Bouse et al., (1990) stated agricultural nozzles generally produce droplets ranging from 10 (extremely fine) to $1000 \mu \mathrm{m}$ (ultra coarse). Understanding the behavior of spray droplets with different diameters is important as a $100 \mu \mathrm{m}$ (very fine) droplet can travel 7.5 times further off target compared to a $500 \mu \mathrm{m}$ (very coarse) droplet given a $5 \mathrm{~km} \mathrm{~h}^{-1}$ wind speed (Creech et al., 2015; Bode, 1987).

Theoretically, nozzles that produce smaller spray droplets increase the efficacy of non-systemic (contact) herbicides. Atomization of spray solution into smaller droplets results in greater coverage of the target tissue, thus maximizing herbicide activity. Rogers and Maki (1986) reported that smaller spray droplets provide greater spray deposition when compared to larger droplets. Additionally, larger spray droplets have greater kinetic 
energy and velocity, which decreases adhesion and increases droplet bouncing and shattering (Spillman, 1984). Shaw et al., (2000) indicated that acifluorfen applied with $250 \mu \mathrm{m}$ (medium) spray droplets provided the greatest common cocklebur (Xanthium strumarium L.) control. Reduced glufosinate and paraquat control of broadleaf signalgrass [Urochloa platyphylla (Munro ex. C. Wright) R. D. Webster] and common cocklebur has been observed as droplet size increased (Etheridge et al., 2001). In contrast, Uremis et al., (2004) and Berger et al., (2014) reported similar efficacy from different size spray droplets of acifluorfen and lactofen, respectively. The convoluted results found in the literature regarding efficacy of contact herbicides could be attributed to specific relationships between biotic and abiotic factors such as plant species, population genetics and density, climate, and soil type. Chachalis et al., (2001) reported reduced spray droplet contact using acifluorfen on ivyleaf morningglory (Ipomoea hederacea Jacq.) compared to other three morningglory species.

The development of digital application technology has allowed pulse width modulation (PWM) systems to be implemented into agricultural sprayers (Bode and Bretthauer, 2007). Pulse width modulation (PWM) sprayers increase application flexibility as this system maintains pressure and spray droplet size constant at different driving speeds while variably controlling flow (Butts et al., 2018). In PWM systems, each spray nozzle is equipped with an electronically-powered solenoid that typically pulses on a frequency of 10 pulses per second. The relative proportion of time each valve remains open is called duty cycle and allows for variable flow rate. In comparison to conventional spray systems, PMW sprayers provide flow rate changes without altering application pressure and nozzle type, allowing the operator to make applications at 
different speeds while maintaining the same droplet size and carrier volume. Previous research has shown that PWM duty cycle has minimum to no effect on spray droplet when using non-venturi nozzles (Butts et al., 2019; Giles et al., 1996). Additionally, when operated at or above $40 \%$ duty cycles, PWM sprayers did not impact spray pattern and coverage (Butts et al., 2019a; Mangus et al., 2017). Thus, PWM sprayers can be used to make spray applications where droplet size is held constant.

The development and spread of Palmer amaranth (Amaranthus palmeri S. Wats.) populations resistant to multiple herbicide modes of action (MOA) has complicated control practices across the southern United States. Palmer amaranth populations resistant to eight different herbicide MOA have been documented; 5-enolpyruvylshikimate-3phosphate synthase (EPSPs) inhibitors, acetolactate synthase (ALS) or acetohydroxyacid synthase (AHAS) inhibitors, photosystem II (PSII) inhibitors, synthetic auxins, 4hydroxyphenylpyruvate dioxygenase (HPPD) inhibitors, microtubule inhibitors, very long chain fatty acid (VLCFA) inhibitors, and prothoporphyrinogen oxidase (PPO) inhibitors (Heap, 2019). Biotypes resistant to PPO-inhibiting herbicides (fomesafen) were first documented in Arkansas in 2011. Consequently, populations resistant to acifluorfen and lactofen were reported in 2016 (Heap, 2019). Research has shown that Palmer amaranth populations from fields located in the northern Mississippi Alluvial Valley region are likely to be resistant to PPO-inhibiting herbicides (Bond et al., 2016). This scenario has caused concern as acifluorfen is an important postemergence option for growers to control Palmer amaranth in soybean [Glycine max (L.) Merr.], peanut (Arachis hypogaea L.), and rice (Oryza sativa L.) (Sweat et al., 1998). Given the rapid spread of PPO-resistant Palmer amaranth, cost effective means of application that 
maximize acifluorfen effectiveness for Palmer amaranth control and mitigate spray drift are needed. Hence, the objectives of this experiment were to evaluate the influence of droplet size on acifluorfen efficacy on Palmer amaranth control and develop prediction models to optimize spray droplet size for Palmer amaranth control.

\subsection{Materials and Methods}

Experiments were conducted in 2016, 2017, and 2018 in a non-crop environment in Dundee, MS (2016-2018) on a Sharkey clay soil, Beaver City, NE (2016-2017) on a Holdrege silt loam, and Robinsonville, MS (2017-2018) on a Commerce silt loam to evaluate the effect of acifluorfen spray droplet size for Palmer amaranth control. Acifluorfen (Ultra Blazer ${ }^{\circledR}$, UPL Corporate, King of Prussia, PA 19406) at $0.42 \mathrm{~kg}$ ai ha ${ }^{-1}$ plus crop oil concentrate (Agri-Dex ${ }^{\circledR}$, Helena Chemical Co., Collierville, TN 38017) at $1 \% \mathrm{v} / \mathrm{v}$ was applied to $15 \mathrm{~cm}$ tall Palmer amaranth. Treatments consisted of six targeted droplet sizes $(150,300,450,600,750$, and $900 \mu \mathrm{m})$ determined from the volume median diameter $\left(D_{v 0.5}\right)$ of the measured droplet size distribution. One nontreated control at each location was used for treatment comparison. Plot dimension was 4 meters wide by 12 meters long, and treatments were arranged in a randomized complete block design with four replications. Treatments were applied using a tractor mounted sprayer equipped with a Pin Point ${ }^{\circledR}$ pulse width modulation (PWM) system (Capstan Ag Systems, Inc., Topeka, KA 66609) using non-venturi Wilger ${ }^{\mathrm{TM}}$ precision spray tips (Wilger Inc., Lexington, TN 38351 ) operated at $4.8 \mathrm{~km} \mathrm{~h}^{-1}$ and spray volume of $140 \mathrm{~L} \mathrm{ha}^{-1}$. Individual site-year information including GPS coordinates, application date, and weather conditions at the time of application are presented in Table 2.1. 
Prior to experiment initiation, acifluorfen droplet size spectra was characterized in a low-speed wind tunnel located at the Pesticide Application Technology (PAT) Laboratory in North Platte, Nebraska (Table 2.2). Nozzle type, orifice size, and application pressure necessary to produce the aforementioned droplet sizes were determined using a Sympatec HELOS-VARIO/KR laser diffraction system (Sympatec Inc., Clausthal-Zellerfeld, Germany 38678) equipped with R7 lens capable of detecting particle sizes ranging from 18 to $3500 \mu \mathrm{m}$. Procedures for actual droplet size determination follow those described by Butts et al., (2018). Droplet size classifications were assigned in accordance with ASABE S572.1 (ASABE, 2009).

\subsubsection{Data Collection}

Visual evaluation of Palmer amaranth control was collected at 7, 14, 21, and 28 days after herbicide treatment (DAT). Palmer amaranth control was evaluated on a scale of 0 (no control) to $100 \%$ (complete death of all plants) relative to the nontreated check (Frans et al., 1986). Prior to herbicide application, 10 plants per plot measuring $15 \mathrm{~cm}$ in height were tagged at the soil surface for future above ground dry biomass evaluation. At the end of the experiment, tagged plants were harvested, placed in paper bags, removed from experimental area, and dried in a forced air dryer at $55^{\circ} \mathrm{C}$ for 72 hours. Tagged plants were also used to determine visual weed control in plots where new emergence or regrowth occurred.

\subsubsection{Statistical Analysis}

Generalized additive model (GAM) analysis was conducted in R x64 3.4.3 using the $m g c v$ package to provide estimated optimum droplet size for Palmer amaranth control 
(Crawley, 2013). Data from 28 DAT were used to model Palmer amaranth visual control and dry biomass reduction estimation. The nontreated was included in the experiment for comparison but was not included in GAM analysis for either response variable to allow better separation between droplet sizes. In order to meet model assumptions, visual Palmer amaranth control data was converted to a beta distribution. Visual Palmer amaranth control data was bound between 0 and 1 to reduce distribution error. Palmer amaranth dry biomass reduction was subjected to a natural log-transformation to reduce data skewness and increase normalization. Dry biomass data were then back transformed for clearer effect interpretation. Response variables were visual Palmer amaranth control and dry aboveground biomass. Response variables were subjected to one smooth variable (droplet size) as described by Butts et al. (2018) (Equation 2.1).

$$
\text { Response variable } \sim \text { s(Target droplet size })
$$

Models were used to predict optimal droplet size that maximized Palmer amaranth control and dry biomass reduction. In addition, predicted values were used to calculate the droplet size range in which $90 \%$ maximum weed control was maintained.

\subsection{Results and Discussion}

Generalized additive model analysis for visual Palmer amaranth control and dry biomass across seven site-years are presented in Figure 2.1. Smooth term estimated degrees of freedom (EDF) and deviance explained values are given in Table 2.3. A smooth term EDF of one indicates minimum model fluctuation and characterizes a linear model (Butts et al., 2018). Deviance explained values provide a model fitting estimation between predicted values and actual observations, with greater percentages representing an overall better model fit. 
Smooth term EDF values of one were observed for both response variables (Table 2.3) (Figure 2.1). A deviance explained of $7.23 \%$ was observed for visual Palmer amaranth control, meaning $7.23 \%$ of variation in visual Palmer amaranth control is due to spray droplet size. In terms of Palmer amaranth dry biomass, $0.004 \%$ deviance explained was observed indicating that control from differing spray droplet size was not a good predictor of Palmer amaranth dry biomass. Similar results have been reported by Butts $e t$ al., (2018). Weather conditions, geographic location, soil type, fertility levels, weed density, and population genetics should be investigated in future research to implement acifluorfen model assumptions across multiple locations.

Generalized additive model predicted increased visual Palmer amaranth control and dry biomass reduction following application with finer spray droplets. Models suggest that maximum visual Palmer amaranth control and dry biomass reduction could be achieved with $150 \mu \mathrm{m}$ (fine) droplets (Table 2.4). These results agree with the general hypotheses that smaller droplets provide greater coverage, thus increasing the efficacy of contact herbicides. Rogers and Maki (1986) reported increased acifluorfen deposition when droplet size was reduced from 410 (very coarse) to $130 \mu \mathrm{m}$ (fine). Similar research reported that the use of $100 \mu \mathrm{m}$ (very fine) spray droplets increased herbicide phytotoxicity (McKinlay et al., 1972; Prasad et al., 1987). According to prediction models, droplet sizes ranging from 150 (fine) to $425 \mu \mathrm{m}$ (very coarse) could be used to maintain at least $90 \%$ maximum Palmer amaranth control. These observations suggest that fine, medium, coarse, and very coarse droplets may be used without loss of Palmer amaranth control and larger spray droplets could be implemented as a spray drift mitigation practice. 
Although finer droplets provided greater Palmer amaranth dry biomass reduction, results suggest that any droplet size between 150 (fine) and $900 \mu \mathrm{m}$ (ultra coarse) could be used to maintain at least $90 \%$ of maximum dry biomass reduction (Table 2.4). The small deviance explained values $(7.23 \%$ and $0.004 \%)$ indicate large variability in visual Palmer amaranth control and dry biomass in response to spray droplet size. Data from GAM analysis across all site-years suggest that fine, medium, coarse, and very coarse droplet sizes can be used to maintain at least $90 \%$ of maximum Palmer amaranth control. For areas in close proximity to susceptible crops, the use of very coarse droplets is recommended. However, spray droplet size recommendations across a wide range of geographic areas should be made with caution. The low deviance explained values obtained from the GAM analysis across all site-years highlight the importance of location-specific recommendations.

\subsubsection{Acifluorfen location-specific analysis}

Location-specific analyses were conducted to minimize discrepancies between model predictions and observed values across all site-years. Generalized additive models for visual Palmer amaranth control and dry biomass for Dundee, MS, Beaver City, NE, and Robinsonville, MS across years are presented in Figures 2.2, 2.3, and 2.4, respectively. Generalized additive model smooth term EDF and deviance explained values for each site pooled across years are presented in Table 2.5.

Smooth term EDF values of 4.85 and 3.29 indicate a non-linear characterization of Palmer amaranth control in Dundee, MS and Robinsonville, MS, respectively (Table 2.5) (Figures 2.2, 2.4). Location-specific analysis for these locations significantly increased deviance explained to 49.6\% (7-fold increase) in Dundee, MS and 20\% (3-fold 
increase) in Robinsonville, MS, which indicates better model performance in predicting Palmer amaranth control as influenced by spray droplet size. Unlike previous models, GAM model for visual weed control in Beaver City, NE pooled over two years had a linear pattern (smooth term EDF $=1)$ and slightly increased deviance explained $(8.85 \%)$ when compared to prediction model developed across all site-years (7.23\%) (Table 2.5) (Figure 2.3). The small deviance explained increase (1.62\%) observed in Beaver City, NE may be a result of weed density differences observed at this site between 2016 and 2017 . Although experimental areas were in near proximity, Palmer amaranth density was significantly lower in 2017 compared to 2016 (Table 2.1).

Prediction models developed using Palmer amaranth dry biomass had deviance explained values of $1.76 \%$ for Dundee, MS and $0.35 \%$ for Beaver City, NE, and Robinsonville, MS, (Table 2.5). Therefore, spray droplets to achieve maximum weed control and 90\% maximum weed control were calculated using visual Palmer amaranth control prediction models (Table 2.6). Generalized additive model analysis indicates that droplet size recommendation should be location-specific. Prediction models for Dundee, MS using observations pooled over three years suggest that maximum Palmer amaranth visual control could be achieved with $250 \mu$ m (medium) droplets. Previous research conducted by De Cock et al., (2017) reported that spray droplets between $200 \mu \mathrm{m}$ (fine) and $250 \mu \mathrm{m}$ (medium) provided moderate kinetic energy and drift reduction, leading to increased spray deposition. Additionally, $80 \%$ spray deposition was observed following application with $250 \mu \mathrm{m}$ (medium) droplets. Shaw et al., (2000) observed greater herbicide efficacy when acifluorfen was applied with $250 \mu \mathrm{m}$ (medium) droplets. Differently, weed control models for Beaver City, NE and Robinsonville, MS suggest 
150 (fine) and $370 \mu \mathrm{m}$ (coarse) droplets, respectively, could be used to achieve maximum Palmer amaranth control (Table 2.6).

Generalized additive model analysis indicates that fine and medium spray droplets can be used to achieve $90 \%$ or greater weed control in Dundee, MS [180 (fine) to $310 \mu \mathrm{m}$ (medium) ] and Beaver City, NE [150 (fine) to $340 \mu \mathrm{m}$ (medium)] (Table 2.6). In addition, a broader droplet size range could be used to maintain $90 \%$ of maximum Palmer amaranth control in Robinsonville, MS [220 (medium) to $680 \mu \mathrm{m}$ (ultra coarse)] allowing for application with larger spray droplets that may reduce spray drift (Table 2.6).

\subsubsection{Acifluorfen site-year analysis}

An increase in model accuracy was observed when each site-year was analyzed independently. Smooth term EDF values and deviance explained for all site-years are shown in Table 2.7. Generalized additive models for Dundee, MS in 2016 and 2017 were highly accurate in predicting visual Palmer amaranth control. According to deviance explained values, 77.2 and $76.8 \%$ of differences observed in Palmer amaranth control in 2016 and 2017 could be explained by droplet size (Table 2.7). Smooth term EDF values greater than one represent the complexity of these models. Conversely, the 2018 GAM model showed a reduced deviance explained value and a linear pattern. These differences could be correlated to the increased temperature and relative humidity recorded at the time of application in 2018 (Table 2.1). Similar differences were observed in Robinsonville, MS. However, greater prediction accuracy was observed in 2018 (deviance explained $=56.30 \%$ ). Although model trends for Beaver City, NE were similar in 2016 and 2017, GAM analysis provided greater visual Palmer amaranth control prediction accuracy in 2016. As mentioned previously, differences in population density 
at experimental areas in Beaver City, NE between 2016 and 2017 could contribute to discrepancies in deviance explained (Table 2.1).

Droplet size recommendations for maximum Palmer amaranth control and 90\% of maximum control are presented in Table 2.8. Analysis by year suggests $150 \mu \mathrm{m}$ (fine) and $250 \mu \mathrm{m}$ (medium) droplets could be used to achieve maximum Palmer amaranth control in Dundee, MS, in 2016 and 2017, respectively. In addition, fine and medium size droplets could be used to maintain at least $90 \%$ of maximum control. Prediction using individual location observations from 2018 suggest the use of very coarse droplets (490 $\mu \mathrm{m})$ could have be used to maintain $90 \%$ of maximum weed control. However, it is important to note the level of discrepancy between predicted values and actual observations in 2018 (deviance explained $=18.20 \%)$. Hence, the recommendation of fine and medium spray droplets to maximize Palmer amaranth control in Dundee, MS could be made based on stronger model assumptions. Individual GAM models that had greater deviance explained values indicated that greatest Palmer amaranth control is achieved with $310 \mu \mathrm{m}$ (medium) and $360 \mu \mathrm{m}$ (coarse) spray droplets in Beaver City, NE and Robinsonville, MS, respectively (Table 2.8). Furthermore, 90\% of maximum control could be maintained with droplets ranging from medium to very coarse in Robinsonville, and fine to extremely coarse in Beaver City, NE.

The use of GAM models to determine optimal droplet size prediction is locationspecific. Disparities observed across site-years could be a result of different weather conditions, fertility levels, light incidence, and weed density across geographies. In addition to the complex interactions between biotic and abiotic factors across different environments, the use of PWM sprayers is essential to location-specific weed 
management practices. Future research should investigate the influence of environmental and agronomic factors on model prediction in order to improve pesticide application efficiency.

\subsection{Conclusion}

Prediction models developed across years and a wide geographic area suggest that acifluorfen applied with $150 \mu \mathrm{m}$ (fine) spray droplets may be used to optimize Palmer amaranth control. Nevertheless, a location-specific approach substantially increased model prediction accuracy. Generalized additive model analysis indicates that acifluorfen application efficacy for Palmer amaranth control in Dundee, MS is maximized using 250 $\mu \mathrm{m}$ (medium) spray droplets. Furthermore, $150 \mu \mathrm{m}$ (fine) and $370 \mu \mathrm{m}$ (coarse) spray droplets could be used to optimize acifluorfen effectiveness for Palmer amaranth control in Beaver City, NE and Robinsonville, MS, respectively. Fine and medium spray droplets are recommended to maintain at least $90 \%$ of maximum Palmer amaranth control in Dundee, MS and Beaver City, NE. In addition, coarse, very coarse, and extremely coarse spray droplets could be used to achieve $90 \%$ of maximum Palmer amaranth control in

Robinsonville, MS. The level of interaction between biotic and abiotic factors is complex and varies across locations. Therefore, prediction models should be created using location-specific observations to strengthen weed control management practices that optimize herbicide efficacy and mitigate spray drift. Further investigation of weather conditions, soil type, growth stage, population genetics, and resistance levels will improve droplet size prediction models across multiple geographies. 
Table 2.1 Year, location, GPS coordinates, Palmer amaranth density, application date, and weather conditions at the time of herbicide application.

\begin{tabular}{|c|c|c|c|c|c|c|c|}
\hline \multirow[b]{2}{*}{ Year } & \multirow[b]{2}{*}{ Location } & \multirow[b]{2}{*}{ GPS Coordinates } & \multirow[b]{2}{*}{$\begin{array}{l}\text { AMAPA } \\
\text { Density }^{\mathrm{a}}\end{array}$} & \multirow[b]{2}{*}{ Date } & \multicolumn{3}{|c|}{ Application weather conditions } \\
\hline & & & & & Wind speed & Air temperature & $\begin{array}{l}\text { Relative } \\
\text { humidity }\end{array}$ \\
\hline & & & Plants $\mathrm{m}^{-2}$ & & $\mathrm{~km} \mathrm{~h}^{-1}$ & ${ }^{\circ} \mathrm{C}$ & $\%$ \\
\hline 2016 & Dundee, MS & $\begin{array}{l}34^{\circ} 32^{\prime} 39^{\prime \prime} \mathrm{N} \\
90^{\circ} 28^{\prime} 22^{\prime \prime} \mathrm{W}\end{array}$ & 140 & 08 Sep. & 5 & 21 & 40 \\
\hline 2016 & Beaver City, NE & $\begin{array}{c}40^{\circ} 14^{\prime} 2^{\prime \prime} \mathrm{N} \\
98^{\circ} 57^{\prime} 10^{\prime \prime} \mathrm{W}\end{array}$ & 100 & 07 June & 6 & 22 & 40 \\
\hline 2017 & Dundee, MS & $\begin{array}{l}34^{\circ} 32^{\prime} 39^{\prime \prime} \mathrm{N} \\
90^{\circ} 28^{\prime} 22^{\prime \prime} \mathrm{W}\end{array}$ & 334 & 01 June & 14 & 25 & 74 \\
\hline 2017 & Beaver City, NE & $\begin{array}{c}40^{\circ} 14^{\prime} 2^{\prime \prime} \mathrm{N} \\
98^{\circ} 57^{\prime} 10^{\prime \prime} \mathrm{W}\end{array}$ & 25 & 22 Aug. & 10 & 23 & 55 \\
\hline 2017 & Robinsonville, MS & $\begin{array}{l}34^{\circ} 49^{\prime} 41^{\prime \prime} \mathrm{N} \\
90^{\circ} 17^{\prime} 21^{\prime \prime} \mathrm{W}\end{array}$ & 288 & 01 June & 5 & 29 & 60 \\
\hline 2018 & Dundee, MS & $\begin{array}{l}34^{\circ} 32^{\prime} 39^{\prime \prime} \mathrm{N} \\
90^{\circ} 28^{\prime} 22^{\prime \prime} \mathrm{W}\end{array}$ & 217 & 25 June & 16 & 32 & 82 \\
\hline 2018 & Robinsonville, MS & $\begin{array}{l}34^{\circ} 49^{\prime} 41^{\prime \prime} \mathrm{N} \\
90^{\circ} 17^{\prime} 21^{\prime \prime} \mathrm{W}\end{array}$ & 200 & 15 June & 2 & 33 & 51 \\
\hline
\end{tabular}

${ }^{a}$ Palmer amaranth (AMAPA) population density (plants $\mathrm{m}^{-2}$ ) collected one week prior herbicide application date. 
Table 2.2 Herbicide, nozzle type, application pressure and droplet size classification for acifluorfen droplet size treatments.

\begin{tabular}{lcccccc}
\hline Herbicide & Nozzle $^{\mathrm{a}}$ & $\begin{array}{c}\text { Application } \\
\text { pressure }\end{array}$ & $\begin{array}{c}\text { Target } \\
\text { droplet } \\
\text { size }^{\mathrm{b}}\end{array}$ & $\begin{array}{c}\text { Actual } \\
\text { droplet } \\
\text { size }\end{array}$ & $\begin{array}{c}\text { Standard } \\
\text { error }\end{array}$ & $\begin{array}{c}\text { Spray } \\
\text { classification }^{\mathrm{c}}\end{array}$ \\
\hline \multirow{5}{*}{ Acifluorfen } & ER 110015 & 414 & 150 & 153 & 0.60 & $\mathrm{~F}$ \\
& SR 11004 & 324 & 300 & 300 & 3.58 & $\mathrm{M}$ \\
& DR 11003 & 414 & 450 & 453 & 0.98 & VC \\
& DR11006 & 331 & 600 & 597 & 0.73 & EC \\
& UR11006 & 345 & 750 & 746 & 1.95 & UC \\
& UR 11010 & 276 & 900 & 904 & 3.46 & UC \\
\hline
\end{tabular}

${ }^{\mathrm{a}}$ Flat fan, non-venturi nozzles; Wilger ${ }^{\mathrm{TM}}$ precision spray tips (Wilger Inc., Lexington, TN 38351).

${ }^{\mathrm{b}}$ Target droplet sizes were used in data analysis.

${ }^{\mathrm{c}}$ Spray classification according to ASABE S572.1 where F=Fine, M=Medium, VC=Very Coarse, EC=Extremely Coarse, and UC=Ultra Coarse.

Table 2.3 Generalized additive model (GAM) smooth parameters and deviance explained for visual Palmer amaranth control and dry biomass reduction.

\begin{tabular}{lcc}
\hline Response variable $^{\mathrm{a}}$ & Smooth term EDF $^{\mathrm{b}}$ & Deviance explained $^{\mathrm{c}}$ \\
\hline Visual control & 1 & $\%$ \\
Dry biomass & 1 & 7.230 \\
\hline
\end{tabular}

${ }^{a}$ Visual Palmer amaranth control and dry biomass reduction data were pooled across seven site years.

${ }^{\mathrm{b}}$ Smooth term estimated degrees of freedom (EDF) provides an estimation of model fluctuation for a response variable. Smooth term estimated degrees of freedom (EDF) values of 1 represent a linear model.

${ }^{\mathrm{c}}$ Deviance explained value represents the variability of a given response variable due to droplet size. 
Table 2.4 Generalized additive model (GAM) analysis for maximum visual Palmer amaranth control and dry biomass pooled across all site-years.

\begin{tabular}{|c|c|c|c|c|}
\hline \multirow[b]{2}{*}{ Response variable } & \multicolumn{4}{|c|}{ Droplet size } \\
\hline & $\begin{array}{c}\text { Maximum } \\
\text { weed control }^{\mathrm{a}}\end{array}$ & $\begin{array}{c}\text { Spray } \\
\text { classification }^{\mathrm{b}}\end{array}$ & $\begin{array}{c}90 \% \text { of } \\
\text { maximum } \\
\text { weed control }^{\mathrm{a}}\end{array}$ & $\begin{array}{c}\text { Spray } \\
\text { classification }^{\mathrm{b}}\end{array}$ \\
\hline & $\mu \mathrm{m}$ & & $\mu \mathrm{m}$ & \\
\hline Visual control & 150 & $\mathrm{~F}$ & 425 & $\mathrm{VC}$ \\
\hline Dry biomass & 150 & $\mathrm{~F}$ & 900 & $\mathrm{UC}$ \\
\hline
\end{tabular}

${ }^{a}$ Droplet sizes required to achieve designated parameters.

${ }^{\mathrm{b}}$ Spray classification according to ASABE S572.2 where F=Fine, M=Medium, VC=Very Coarse, EC=Extremely Coarse, and UC=Ultra Coarse.

Table 2.5 Generalized additive model (GAM) analysis for Palmer amaranth visual control and dry biomass for each site pooled over years.

\begin{tabular}{lcccc}
\hline Site & Year & Response variable & $\begin{array}{c}\text { Smooth term } \\
\text { EDF }^{\mathrm{a}}\end{array}$ & $\begin{array}{c}\text { Deviance } \\
\text { explained }^{\mathrm{b}}\end{array}$ \\
\hline Dundee, MS & $\begin{array}{c}\text { 2016, 2017, } \\
2018\end{array}$ & Visual control & 4.85 & $\%$ \\
& & Dry biomass & 1 & 49.6 \\
Beaver City, NE & 2016,2017 & $\begin{array}{c}\text { Visual control } \\
\text { Dry biomass }\end{array}$ & 1 & 1.76 \\
& & 1 & 8.85 \\
Robinsonville, MS & 2017,2018 & $\begin{array}{c}\text { Visual control } \\
\text { Dry biomass }\end{array}$ & 3.29 & 0.35 \\
& & 1 & 20 \\
\hline
\end{tabular}

${ }^{a}$ Smooth term estimated degrees of freedom (EDF) provides an estimation of model fluctuation for a response variable. Smooth term estimated degrees of freedom (EDF) values of 1 represent a linear model.

${ }^{\mathrm{b}}$ Deviance explained value represents the variability of a given response variable due to droplet size. 
Table 2.6 Droplet size prediction based on generalized additive model (GAM) analysis to reach and maintain $90 \%$ of maximum Palmer amaranth control in Dundee, MS, Beaver City, NE, and Robinsonville, MS pooled across years.

\begin{tabular}{|c|c|c|c|c|c|}
\hline \multirow[b]{2}{*}{ Site } & \multirow[b]{2}{*}{ Year } & \multicolumn{4}{|c|}{ Droplet size } \\
\hline & & $\begin{array}{l}\text { Maximum weed } \\
\text { control }^{\mathrm{a}}\end{array}$ & $\begin{array}{c}\text { Spray } \\
\text { classification }^{\mathrm{c}}\end{array}$ & $\begin{array}{l}90 \% \text { of maximum } \\
\text { weed control }^{\mathrm{b}}\end{array}$ & $\begin{array}{c}\text { Spray } \\
\text { classification }^{\mathrm{c}}\end{array}$ \\
\hline Dundee, MS & $2016,2017,2018$ & $\begin{array}{l}\mu \mathrm{m} \\
250\end{array}$ & M & $\begin{array}{c}\mu \mathrm{m} \\
180-310\end{array}$ & $\mathrm{~F}$ and $\mathrm{M}$ \\
\hline Beaver City, NE & 2016, 2017 & 150 & $\mathrm{~F}$ & $150-340$ & $\mathrm{~F}$ and $\mathrm{M}$ \\
\hline Robinsonville, MS & 2017, 2018 & 370 & $\mathrm{C}$ & $220-680$ & $\begin{array}{c}\mathrm{F}, \mathrm{M}, \mathrm{C}, \mathrm{VC}, \mathrm{EC}, \\
\text { and } \mathrm{UC}\end{array}$ \\
\hline
\end{tabular}

${ }^{\mathrm{a}}$ Droplet sizes required to achieve maximum visual Palmer amaranth control.

$\omega{ }^{\mathrm{b}}$ Droplet sizes between these values can be used to maintain $90 \%$ of maximum Palmer amaranth control.

${ }^{\mathrm{c}}$ Spray classification according to ASABE S572.2 where F=Fine, M=Medium, VC=Very Coarse, EC=Extremely Coarse, and UC=Ultra Coarse. 
Table 2.7 Generalized additive model (GAM) analysis for visual Palmer amaranth control for each site-year.

\begin{tabular}{lccc}
\hline Site & Year & Smooth term EDF & $\begin{array}{c}\text { Deviance } \\
\text { explained }^{\mathrm{b}}\end{array}$ \\
\hline \multirow{2}{*}{ Dundee, MS } & 2016 & 4.25 & $\%$ \\
& 2017 & 4.76 & 77.20 \\
& 2018 & 1 & 76.80 \\
Beaver City, NE & 2016 & 1.87 & 18.20 \\
& 2017 & 1.34 & 45 \\
Robinsonville, MS & 2017 & 1 & 16.20 \\
& 2018 & 3.58 & 6.48 \\
\hline
\end{tabular}

${ }^{a}$ Smooth term estimated degrees of freedom (EDF) provides an estimation of model fluctuation for a response variable. Smooth term estimated degrees of freedom (EDF) values of 1 represent a linear model.

${ }^{\mathrm{b}}$ Deviance explained value represents the variability of a given response variable due to droplet size. 
Table 2.8 Droplet size prediction based on generalized additive model (GAM) analysis to reach and maintain $90 \%$ of maximum Palmer amaranth control in Dundee, MS, Beaver City, NE, and Robinsonville, MS for each site-year.

\begin{tabular}{|c|c|c|c|c|c|}
\hline \multirow[b]{2}{*}{ Site } & \multirow[b]{2}{*}{ Year } & \multicolumn{4}{|c|}{ Droplet size } \\
\hline & & $\begin{array}{l}\text { Maximum weed } \\
\text { control }^{\text {a }}\end{array}$ & $\begin{array}{c}\text { Spray } \\
\text { classification }^{\mathrm{c}}\end{array}$ & $\begin{array}{l}90 \% \text { of maximum } \\
\text { weed control }^{\mathrm{b}}\end{array}$ & $\begin{array}{c}\text { Spray } \\
\text { classification }^{\mathrm{c}}\end{array}$ \\
\hline \multirow{4}{*}{ Dundee, MS } & & $\mu \mathrm{m}$ & & $\mu \mathrm{m}$ & \\
\hline & 2016 & 150 & $\mathrm{~F}$ & $150-250$ & $\mathrm{~F}-\mathrm{M}$ \\
\hline & 2017 & 250 & M & $180-310$ & $\mathrm{~F}-\mathrm{M}$ \\
\hline & 2018 & 150 & $\mathrm{~F}$ & $150-490$ & $\mathrm{~F}, \mathrm{M}, \mathrm{C}$, and $\mathrm{VC}$ \\
\hline \multirow[t]{2}{*}{ Beaver City, NE } & 2016 & 310 & M & $150-550$ & $\begin{array}{c}\mathrm{F}, \mathrm{M}, \mathrm{C}, \mathrm{VC} \text {, and } \\
\mathrm{EC}\end{array}$ \\
\hline & 2017 & 150 & $\mathrm{~F}$ & $150-900$ & $\begin{array}{c}\mathrm{F}, \mathrm{M}, \mathrm{C}, \mathrm{VC}, \mathrm{EC}, \\
\text { and } \mathrm{UC}\end{array}$ \\
\hline \multirow[t]{2}{*}{ Robinsonville, MS } & 2017 & 150 & $\mathrm{~F}$ & $150-900$ & $\begin{array}{c}\mathrm{F}, \mathrm{M}, \mathrm{C}, \mathrm{VC}, \mathrm{EC}, \\
\text { and UC }\end{array}$ \\
\hline & 2018 & 360 & $\mathrm{C}$ & $270-490$ & $\mathrm{M}, \mathrm{C}$, and $\mathrm{VC}$ \\
\hline
\end{tabular}

${ }^{a}$ Droplet sizes required to achieve maximum visual Palmer amaranth control.

${ }^{\mathrm{b}}$ Droplet sizes between these values can be used to maintain $90 \%$ of maximum Palmer amaranth control.

${ }^{\mathrm{c}}$ Spray classification according to ASABE S572.2 where F=Fine, M=Medium, VC=Very Coarse, EC=Extremely Coarse, and UC=Ultra Coarse. 

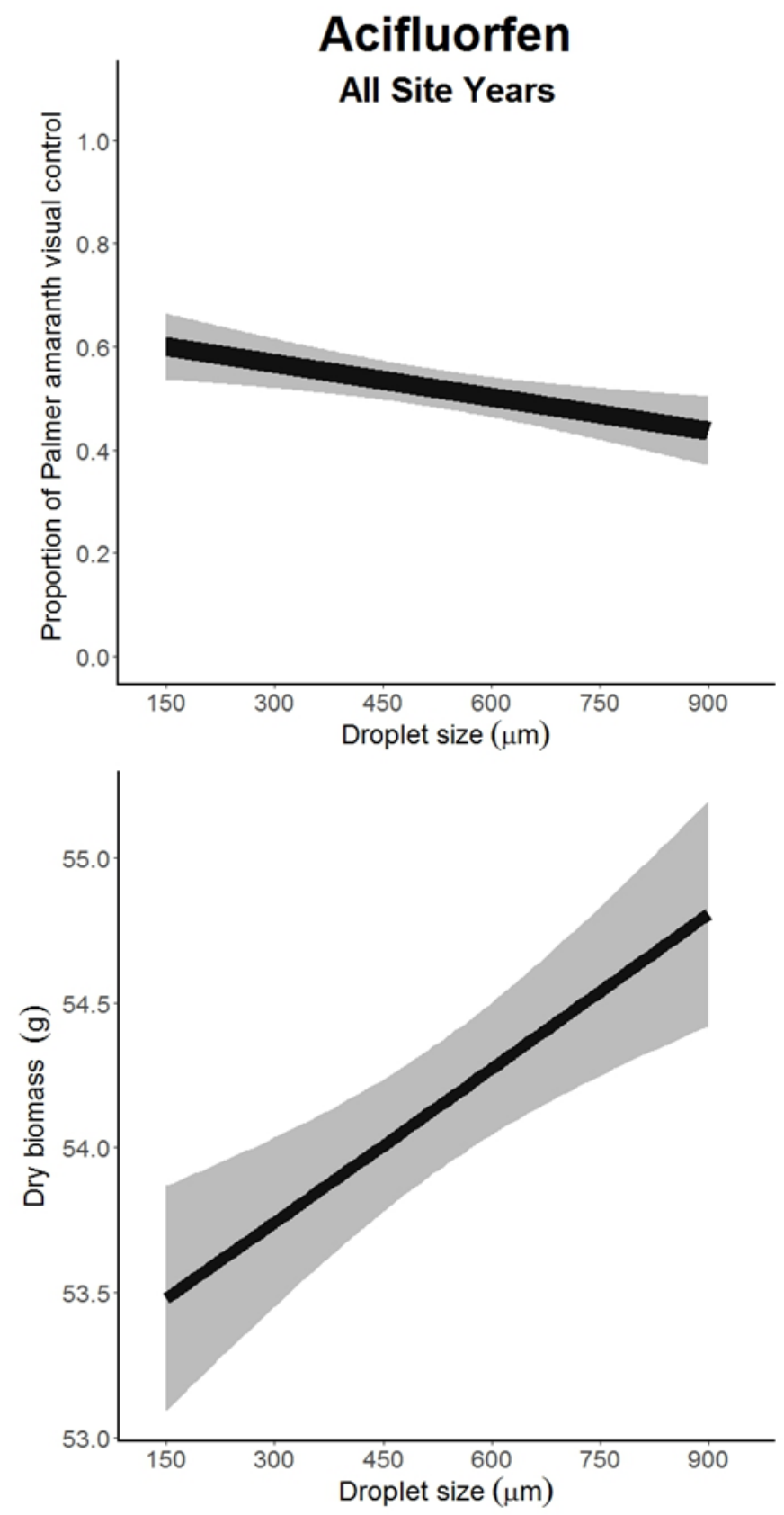

Figure 2.1 Proportion of Palmer amaranth visual control (upper) and dry biomass (lower) 28 days after treatment predicted using generalized additive model (GAM) analysis. The gray shaded area represents the $95 \%$ confidence intervals. 

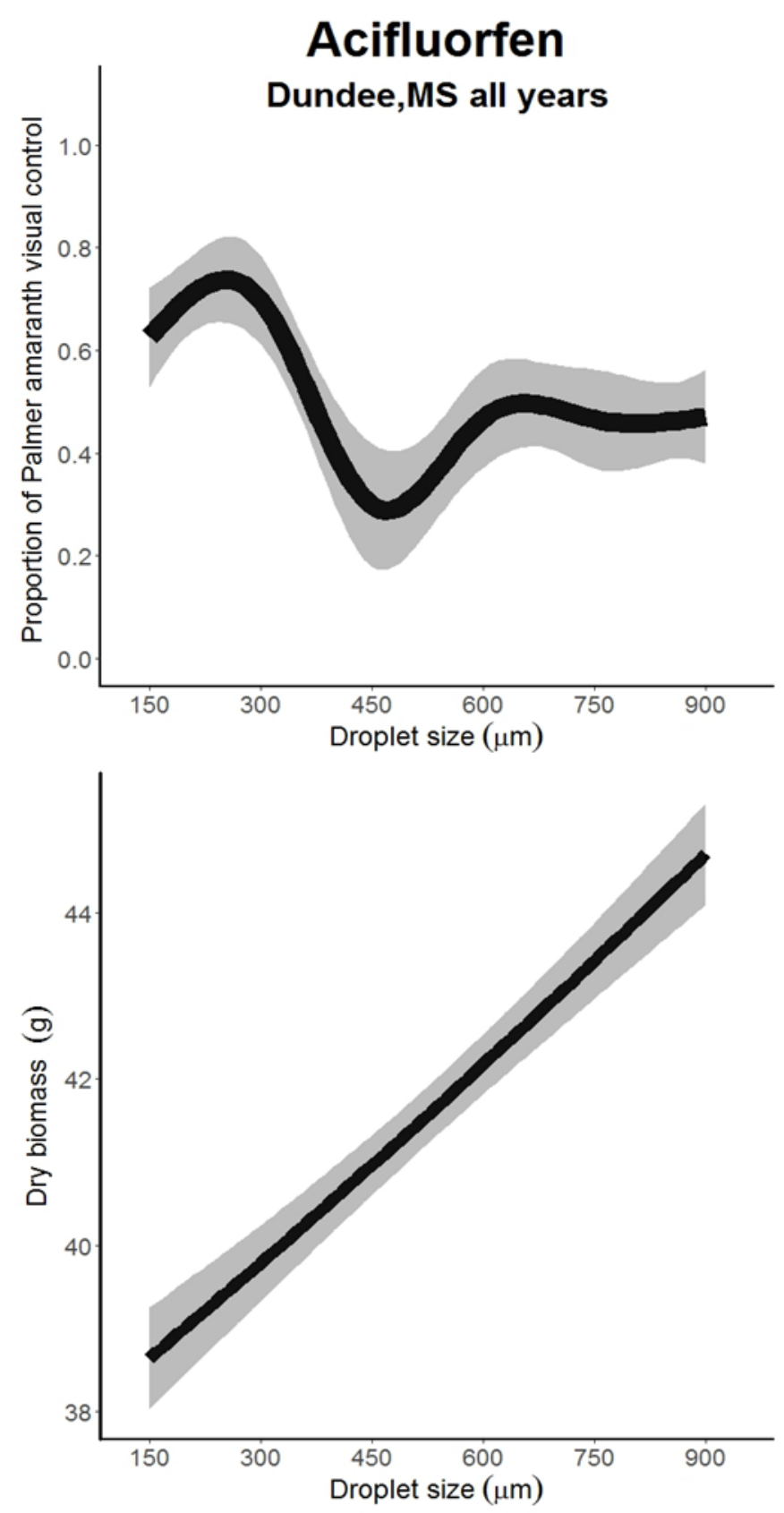

Figure 2.2 Proportion of Palmer amaranth visual control (upper) and dry biomass (lower) 28 days after treatment predicted using generalized additive model (GAM) analysis for Dundee, MS across three site-years. The gray shaded area represents the $95 \%$ confidence intervals. 

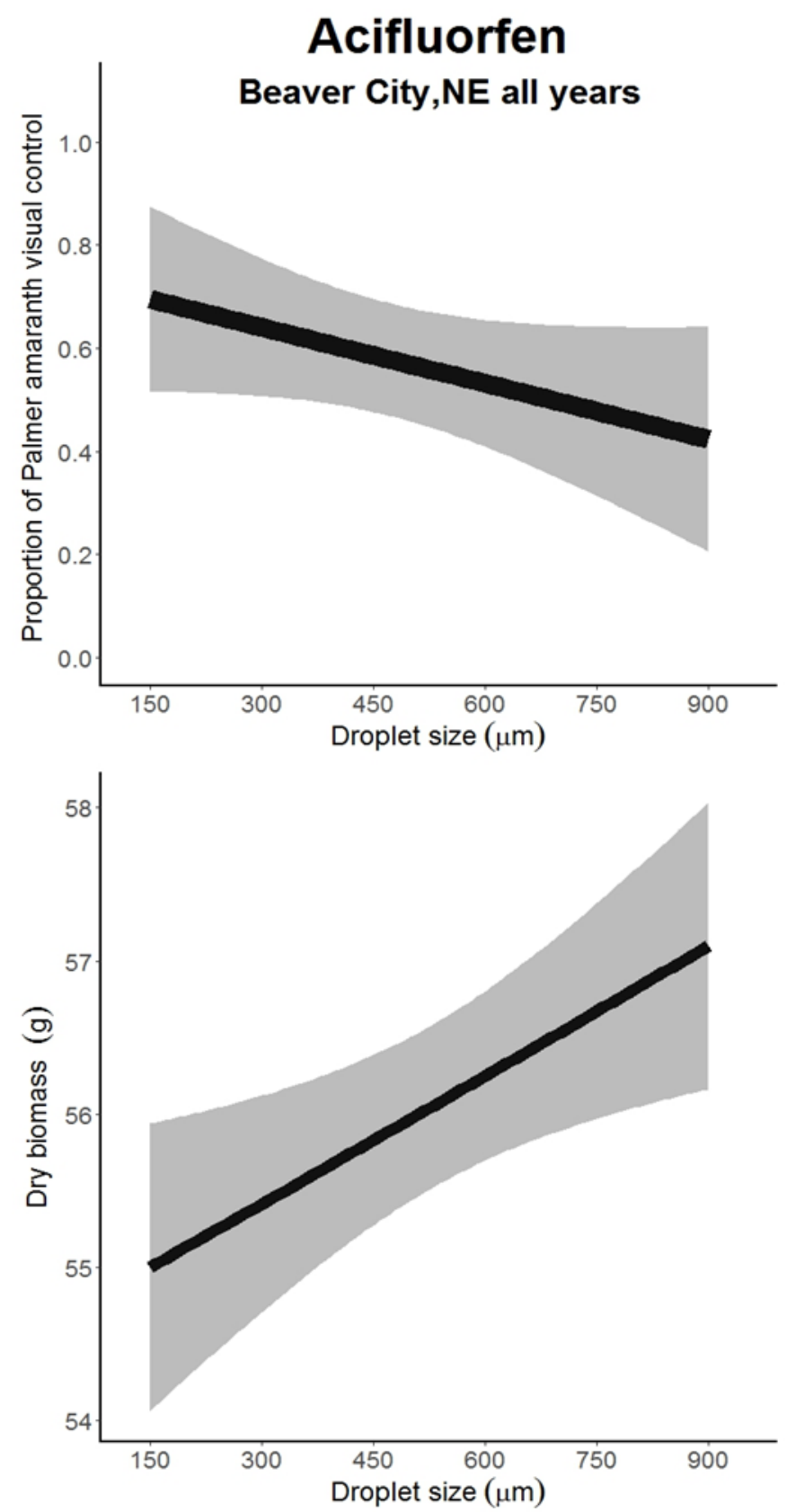

Figure 2.3 Proportion of Palmer amaranth visual control (upper) and dry biomass (lower) 28 days after treatment predicted using generalized additive model (GAM) analysis for Beaver City, NE across two site-years. The gray shaded area represents the $95 \%$ confidence intervals. 

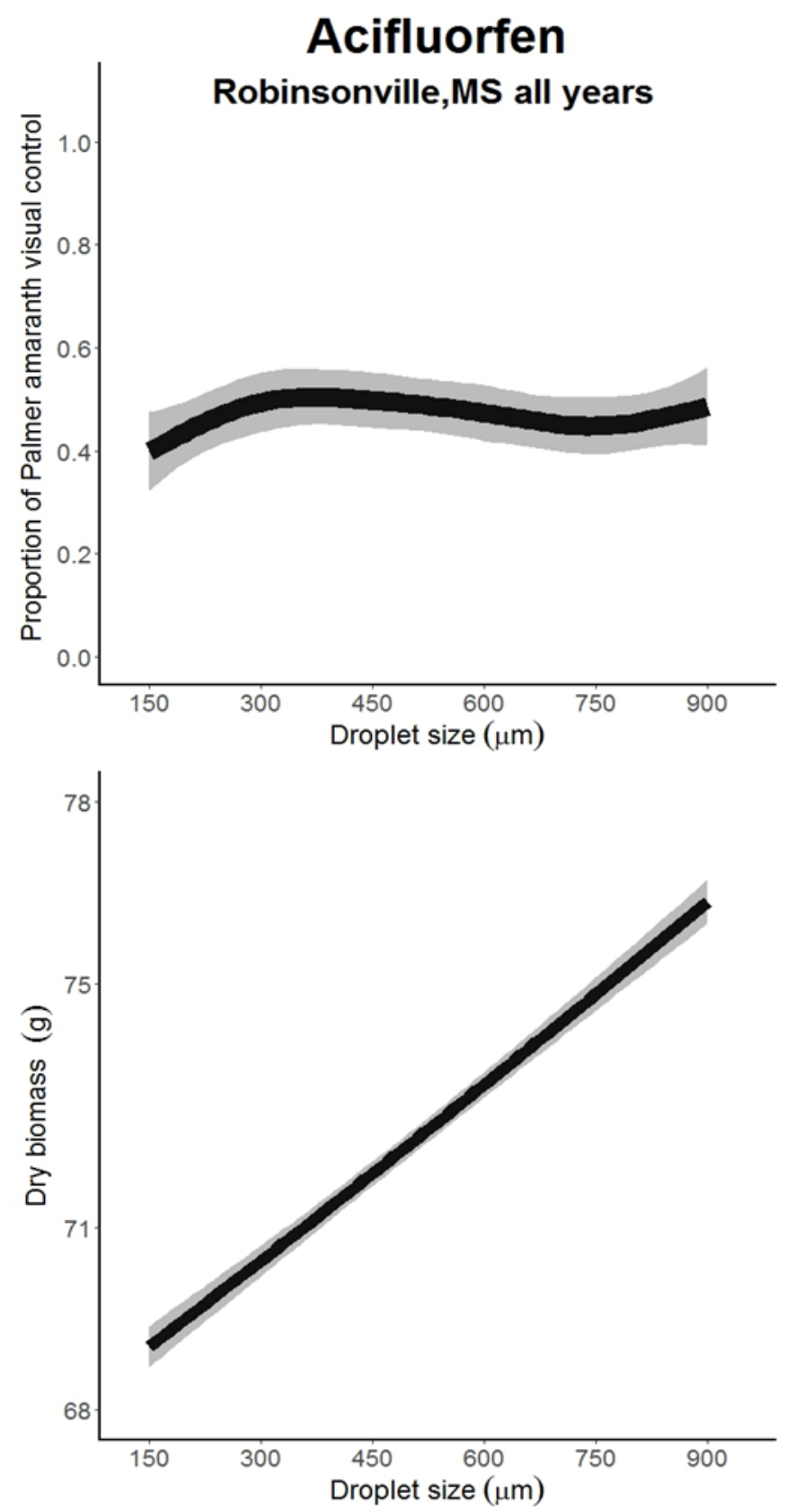

Figure 2.4 Proportion of Palmer amaranth visual control (upper) and dry biomass (lower) 28 days after treatment predicted using generalized additive model (GAM) analysis for Robinsonville, MS across two site-years. The gray shaded area represents the $95 \%$ confidence intervals. 


\subsection{Literature Cited}

[ASABE] American Society of Agricultural and Biological Engineers. 2009. Spray nozzle classification by droplet spectra. St. Joseph, MI: ASABE Standard S572.1. pp. 1-3.

Berger, S.T., Dobrow, M.H., Ferrell, J.A., Webster, T.M. 2014. Influence of carrier volume and nozzle selection on palmer amaranth control. Peanut Sci 41(2):120123.

Beyer Jr, E.M. 1991. Crop protection - meeting the challenge. In Proceedings, Brighton Crop Prot Conf Weeds 1:3-22.

Bode, L. 1987. Spray application technology. In Methods of Applying Herbicides. Champaign, IL. WSSA Monograph 4.

Bode, L.E., Bretthauer, S.M. 2007. Agricultural chemical application technology: a remarkable past and an amazing future. Trans ASABE 51(2):391-395.

Bond, J.A., Reynolds, D.B., Irby, T. 2016. Managing PPO-resistant Palmer amaranth in Mississippi soybean. Mississippi State University Ext. http://www.mississippicrops.com/2016/03/25/managing-ppo-resistant-palmer-amaranth-in-mississippisoybean/ (accessed 14 Jan. 2019)

Bouse, L.F., Kirk, I.W., Bode, L.E. 1990. Effect of spray mixture on droplet size. T ASAE 33(3):783-788.

Butts, T.R., Samples, C.A., Franca, L.X., Dodds, D.M., Reynolds, D.B., Adams, J.W., Zollinger, R.K., Howatt, K.A., Fritz, B.K., Clint Hoffmann, W., Kruger, G.R. 2018. Spray droplet size and carrier volume effect on dicamba and glufosinate efficacy. Pest Manag Sci 74(9):2020-2029.

Butts, T.R., Butts, L.E., Luck, J.D., Fritz, B.K., Hoffmann, W.C., Kruger, G.R. 2019. Droplet size and nozzle tip pressure from a pulse-width modulation sprayer. J Biosyst Eng 178:52-69.

Butts, T.R., Luck, J.D., Fritz, B.K., Hoffmann, W.C., Kruger, G.R. 2019a. Evaluation of spray pattern uniformity using three unique analyses as impacted by nozzle, pressure, and pulse-width modulation duty cycle. Pest Manag Sci., DOI: $10.1002 /$ ps.5352.

Carlsen, S.C.K., Spliid, N.H., Svensmark, B. 2006. Drift of 10 herbicides after tractor spray application. 2. Primary drift (droplet drift). Chemosphere 64(5):778-786. 
Chachalis, D., Reddy, K.N., Elmore, C.D., Steele, M.L. 2001. Herbicide efficacy, leaf structure, and spray droplet contact angle among Ipomoea species and smallflower morning. Weed Sci 49(5):628-634.

Crawley, M.J. 2013. The R Book, Second edn. Chichester, UK Wiley.

Creech, C.F., Henry, R.S., Fritz, B.K., Kruger, G.R. 2015. Influence of herbicide active ingredient, nozzle type, orifice size, spray pressure, and carrier volume rate on spray droplet size characteristics. Weed Technol 29:298-310.

De Cock, N., Massinon, M., Salah, S. O., Lebeau, F. 2017. Investigation on optimal spray properties for ground based agricultural applications using deposition and retention models. J. Biosyst Eng 162:99-111.

Ennis, Jr., Williamson, R.E. 1963. Influence of droplet size on effectiveness of lowvolume herbicidal sprays. Weeds 67-72.

Etheridge, R.E., Hart, W.E., Hayes, R.M., Mueller, T.C. 2001. Effect of venturi-type nozzles and application volume on postemergence herbicide efficacy. Weed Technol 15:75-80.

Frans, R.E., Talbert, R., Marx, D., Crowley, H. 1986. Experimental design and techniques for measuring and analyzing plant responses to weed control practices. p. 37-38. in N.D. Camper (ed.) Research Methods in Weed Science. 3rd ed. Champaign, IL: Southern Weed Science Society.

Giles, D.K., Henderson, G.W., Funk, K. 1996. Digital control of flow rate and spray droplet size from agricultural nozzles for precision chemical application. Precis Agric 729-738.

Heap, I.M. 2019. The International survey of herbicide-resistant weeds. Available at: www.weedscience.org. Accessed: 18 January, 2019.

Knoche, M. 1994. Effect of droplet size and carrier volume on performance of foliageapplied herbicides. Crop Prot 13:163-178.

Mangus, D.L., Sharda, A., Engelhardt, A., Flippo, D., Strasser, R., Luck, J.D., Griffin, T. 2017. Analyzing the nozzle spray fan pattern of an agricultural sprayer using pulse-width modulation technology to generate an on-ground coverage map. Trans ASABE 60:315-325.

McKinlay, K.S., Brandt, S.A., Morse, P., Ashford, R. 1972. Droplet size and phytotoxicity of herbicides. Weed Sci 20:450-452. 
Meyer, C. J., Norsworthy, J. K., Kruger, G. R., Barber, T. 2015. Influence of droplet size on efficacy of the formulated products Engenia ${ }^{\mathrm{TM}}$, Roundup PowerMax ${ }^{\circledR}$, and Liberty®. Weed Technol 29(4):641-652.

Prasad, R. 1987. A study of droplet size and density in relation to efficacy of herbicides. Weed Sci Soc Am Abstr 27:98.

Rogers, R.B., Maki, R. 1986. The Effect of Drop Size on Spray Deposit Efficiency. St. Joseph, MO: American Society of Agricultural Engineering Paper No.86-1508.

Shaw, D.R., Morris, W.H., Webster, E.P., Smith, D.B. 2000. Effects of spray volume and droplet size on herbicide deposition and common cocklebur (Xanthium strumarium) control. Weed Technol 14(2):321-326.

Spillman, J.J. 1984. Spray impaction, retention and adhesion: An introduction to basic characteristics. Pest Manag Sci 15:97-106.

Sweat, J.K., Horak, M.J., Peterson, D.E., Lloyd, R.W., Boyer, J.E. 1998. Herbicide efficacy on four Amaranthus species in soybean (Glycine max). Weed Technol $1: 315-321$.

Taylor, W.A., Womac, A.R., Miller, P.C.H., Taylor, B.P. 2004. An attempt to relate droplet size to drift risk. p. 210-223. In Proceedings of the international conference on pesticide application for drift management. Pullman, WA: Washington State University.

Uremis, I., Bayat, A., Uludag, A., Bozdogan, N., Aksoy, E., Soysal, A., Gonen, O. 2004. Studies on different herbicide application methods in second-crop maize fields. Crop Prot 23(11):1137-1144.

Whisenant, S.G., Bouse, L.F., Crane, R.A., Bovey, R.W. 1993. Droplet size and spray volume effects on honey mesquite mortality with clopyralid. J Range Manag 46:257-261.

Yates, W., Akesson, N., Bayer, D. 1976. Effects of spray adjuvants on drift hazards. Trans Am Soc Agric Eng 19:41-46. 


\section{CHAPTER III}

\section{INFLUENCE OF FLOODING PERIOD AND SEED BURIAL DEPTH ON PALMER AMARANTH (AMARANTHUS PALMERI S. WATS.) SEED GERMINATION}

\subsection{Abstract}

Flooding applied during fall and winter months can provide Palmer amaranth control by reducing seed germination and promoting soil seedbank depletion. Experiments were conducted at the R. R. Foil Plant Science Research Center in Starkville, MS in 2016 and 2017 to evaluate the effect of six flooding periods (noflooding, 1, 2, 3, 4, and 5 months), and two seed burial depths ( 0 and $15 \mathrm{~cm}$ ) across three soil textures (sandy loam, silt, and silt loam) on Palmer amaranth seed damage and germination. Flooding periods of 4 and 5 months resulted in the greatest amount of damaged Palmer amaranth seeds. Furthermore, seed damage was greater when buried in sandy loam soil compared to silt loam soil. An interaction between flooding period and seed burial depth was present for Palmer amaranth seed germination. Palmer amaranth seeds buried at $15 \mathrm{~cm}$ in no-flooding conditions had the greatest total seed germination. Flooding periods of 1 month ( 0 and $15 \mathrm{~cm}$ burial depth) and 2 months $(0 \mathrm{~cm}$ burial depth) provided similar Palmer amaranth seed germination compared to no-flooding $(0 \mathrm{~cm}$ burial depth). Additionally, flooding periods of 3, 4, and 5 months reduced Palmer amaranth seed germination by $10 \%$ (3 and 4 months) and $14 \%$ at $0 \mathrm{~cm}$ burial depth and $36 \%, 40 \%$ and $41 \%$ when seeds were buried at $15 \mathrm{~cm}$, respectively. Moreover, no 
differences in germination were observed due to soil texture. This research demonstrates that flooding for 3,4 , and 5 months is an effective alternative practice to increase soil seedbank depletion and help manage Palmer amaranth populations in sandy loam, silt, and silt loam soils.

\subsection{Introduction}

Palmer amaranth (Amaranthus palmeri $\mathrm{S}$. Wats.) is a $\mathrm{C} 4$, summer annual, dioecious weed specie native to northwestern Mexico, southern California, New Mexico, and Texas (Sauer, 1957). Originated in a xeric environment, Palmer amaranth is naturally opportunistic and adapted for rapid germination and complete life cycle in response to available moisture and temperature (Ehleringer, 1985). Normally, Palmer amaranth flowers during September and October, but decreasing day lengths may hasten flowering process (Keeley et al., 1987). Seeds are smooth, round or disc-shaped, 1 to $2 \mathrm{~mm}$ in diameter, and are usually dispersed by gravity; although, other seed dispersal methods such as irrigation, birds, mammals, plowing, mowing, and harvesting have also been reported (Sauer, 1955; Costea et al., 2004).

Palmer amaranth is an extremely prolific seed producer with one female plant capable of producing up to 600,000 seeds under favorable conditions (Keeley et al., 1987). Although inter- and intraspecific competition may reduce seed production of several plant species, Palmer amaranth seed production remains high in competition with agronomic crops, allowing rapid dissemination of the species (Bond and Oliver, 2006). In

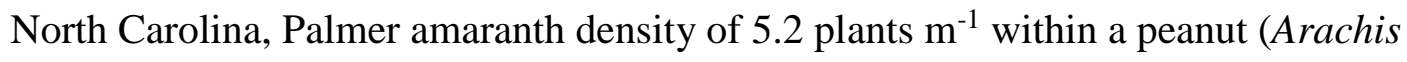
hypogaea L.) row produced 124,000 seeds $\mathrm{m}^{-2}$ (Burke et al., 2007). Research conducted in Georgia reported that Palmer amaranth produced 312,000 seeds per plant when 
competing with cotton and 446,000 seeds per plant in absence of the crop (Webster and Grey, 2015). Additionally, Norsworthy et al., (2014) reported total loss of a cotton field due to Palmer amaranth infestation three years after introduction of the species.

Several factors influence seed germination and dormancy such as soil moisture, temperature, oxygen availability, temperature, light exposure, and microbial activity (Leon et al., 2004). Seed dormancy is also determined by genetic factors and contributes to plant adaptation to a diversity of habitats. When determined by genetic factors, seed dormancy is classified as primary dormancy. Secondary dormancy occurs when unfavorable conditions related to the environment are the determining factor (Graeber et al., 2012). Different classes of seed dormancy have been reported among plant species which can be divided into physiological, morphological, morphophysiological, and combinational dormancy (Baskin and Baskin, 2004; Finch-Savage and Leubner-Metzger, 2006). Physiological and morphological dormancies are the most common mechanisms of weed seed persistence in the soil seedbank (Omani et al., 1999). Previous research reported that differences in Palmer amaranth seed dormancy levels are due to variability in seed physiology and that these differences arose in response to selection pressure such as continuous herbicide applications and tillage practices (Jha et al., 2014; Leon et al., 2006).

Typically, Palmer amaranth emerges from shallow depths and often requires light for breaking dormancy and germination (Baskin and Baskin, 1987; Benech-Arnold et al., 2000; Gallagher and Cardina, 1998). Small-seeded broadleaves such as Palmer amaranth may not survive germination from deeper in the soil profile. As a result, the necessity for light is considered an evolutionary advantage for this type of seed (Pons, 1991). The 
quantity and quality of light reaching the soil surface is deeply dependent on the presence of crop canopy, plant residues, or water. Generally, in the presence of a crop canopy, the light passing through green leaves is filtered and depleted in red light and enriched in farred wavelengths which inhibits germination of many small-seeded broadleaf species (Taylorson and Borthwick, 1969).

Depending on weed species and environmental conditions, seed burial depth can be advantageous for germination and emergence (Forcella et al., 2000). The overlay of soil creates a mulch that sustains high humidity allowing for rapid seed germination. Moreover, the soil protects seeds and seedlings from abnormal air temperatures as well as damage from granivores and herbivores that feed on or near the soil surface (Tolk et al., 1999; Forcella et al., 2000). Sosnoskie et al., (2013) reported that Palmer amaranth seed viability decreased as burial time increased; conversely, seed viability increased with seed burial depth. In a different experiment, Chauhan et al., (2009) observed that spiny amaranth (Amaranthus spinosus L.) and slender amaranth (Amaranthus viridis L.) emergence did not occur when seeds were buried at 4 and $6 \mathrm{~cm}$, respectively.

Flooding is a common practice is most rice (Oryza sativa L.) fields in the United States (Manley, 1999). According to Hardke (2013), 96\% of all rice produced in the United States is grown on silt loam and clay loam soils, and $99.5 \%$ utilizes flooding as part of a weed management program. The presence of water creates an unfavorable environment for most weed species, typically resulting in reduced emergence after permanent flooding is established (Moldenhauer, 2001). Flooding throughout fall and winter months has proven to be an effective practice for rice straw decomposition and waterfowl habitat (Manley et al., 2005). Additionally, fall-winter flooding may reduce 
soybean and rice production costs related to managing rice straw from the previous growing season, winter weeds, and red rice (Oryza sativa L.) (Emory, 1994; Muzzi, 1994). Nevertheless, limited research is available regarding the effects of fall-winter flooding and seed burial depth on Palmer amaranth germination in Mississippi. Therefore, the objective of this experiment was to evaluate the effect of flooding period and seed burial depth on Palmer amaranth seed damage and germination in three different soil textures in Mississippi.

\subsection{Materials and Methods}

An experiment was conducted at the R. R. Foil Plant Science Research Center

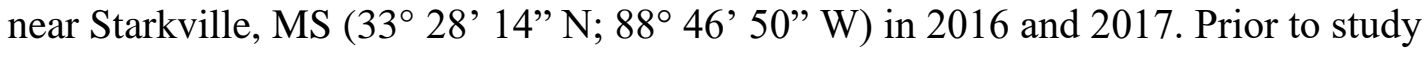
initiation, a germination test was conducted at the Mississippi State Seed Testing Laboratory using Percival ${ }^{\mathrm{TM}}$ model GR41L growth chambers (Percival Scientific, Inc., Perry, IA 50220) to determine germination rate of the selected Palmer amaranth seeds. One hundred Palmer amaranth seeds were placed on moist filter paper inside $18 \mathrm{~cm}$ diameter petri dishes. Day/night temperatures were set at $35 / 30^{\circ} \mathrm{C}$ and $14 / 10$-hour daynight periods. Temperature and light cycles were selected to maximize Palmer amaranth seed germination as described by Guo and Al-Khatib (2003). Germinated seeds were enumerated and removed daily for 15 days. Seeds were considered successfully germinated when the radicle reached $1 \mathrm{~mm}$ in length.

Three soil textures were used in this experiment which included sandy loam (2.8\% clay, $28.4 \%$ silt and $68.8 \%$ sand) from Starkville, MS; silt (2.8\% clay, $84.2 \%$ silt, and $13 \%$ sand) from Stoneville, MS; and silt loam (18\% clay, $64.2 \%$ silt, and $17.8 \%$ sand) from Brooksville, MS. Soils were collected from respective areas, brought to the R. 
R. Foil Plant Science Research Center and allowed to air dry for seven days. Soils were sieved and screened using a 112 Royer Industries ${ }^{\mathrm{TM}}$ soil grinder (Royer Industries Inc., Oshkosh, WI 54903) to eliminate large soil particles. Soils were placed in $27 \mathrm{~L}$ plastic buckets (U-LINE Company, Pleasant Prairie, WI 53158) and buried at $38 \mathrm{~cm}$ depth. Buckets were covered with a plastic mesh to prevent plant residue from falling into the buckets and to protect seeds from damage from small rodents and birds.

To avoid germination of other weed seed present in selected soils, one kg soil samples of each soil texture were collected and autoclaved at $100^{\circ} \mathrm{C}$ for 2 hours using a Market Forge Sterilmatic ${ }^{\circledR}$ autoclave (Market Forge, Burlington, VT 05452). Polyethylene mesh bags measuring $64 \mathrm{~cm}^{2}$ with $500 \mu \mathrm{m}$ pore opening (Elko Filtering Co., Miami, FL 33169) were used for seed storage throughout the duration of the experiment. One hundred Palmer amaranth seeds, and approximately 20 grams of sterilized soil were placed inside each bag. Two polyethylene mesh bags containing seed and soil were placed in each bucket. One bag was buried at $15 \mathrm{~cm}$ depth and the other placed on soil surface. Buckets were flooded with $15 \mathrm{~cm}$ of water above soil surface for one of five flooding periods. Flooding periods were as follows: 5 months (October through February); 4 months (October through January); 3 months (October through December); 2 months (October through November); 1 month (October); and no-flooding (October through February). The experimental design was a split-plot with three replicates. Plots were $3 \mathrm{~m}$ wide and $3 \mathrm{~m}$ long with each bucket placed in the center of each plot (Figure 3.1). Main plot factors were flooding period and soil texture, and subplot factor was seed burial depth. 
Following the completion of each flooding period, polyethylene bags were removed from each bucket and seeds were separated from soil using a $\mathrm{N}^{\circ} 35,500 \mu \mathrm{m}$ mesh sieve (VWR ${ }^{\mathrm{TM}}$, International, Radnor, PA 19087). Under a microscope, Palmer amaranth seeds were enumerated and characterized as normal or damaged. Seeds were classified as damaged when seeds were hollow and/or presenting substantial damage to the seed coat as shown in Figure 3.2. After visual characterization, germination testing was conducted at the Mississippi University State Seed Testing Laboratory. Palmer amaranth seeds were germinated according to the aforementioned procedure described by Guo and Al-Khatib (2003). Seed damage and germination values were analyzed using PROC MIXED procedure in SAS v.9.4 (SAS® Institute Inc., Cary, NC 27513) and means were separated using Fisher's Protected LSD at 0.05 level of significance. Fixed effects consisted of flooding period, soil texture, and burial depth, and random effects were year and replication nested in year.

\subsection{Results and Discussion}

\subsubsection{Palmer amaranth seed damage}

Analysis of variance (ANOVA) for Palmer amaranth seed damage is presented in Table 3.1. The absence of a significant interaction between sources of variability allow for individual analysis of fixed factors. Palmer amaranth seed characterization was influenced by soil texture $(\mathrm{p}=0.0419)$ and flooding period $(\mathrm{p}<0.0001)$. The greatest amount of damaged Palmer amaranth seeds was observed in the sandy loam soil from Starkville (Figure 3.3). Although greater levels of seed damage were observed in the sandy loam soil texture, damage did not differ when seeds were placed in silt soil from Stoneville, MS. Additionally, 5\% less damaged seeds were observed when placed in silt 
loam soil from Brooksville, MS. Differences in seed characterization could be correlated to the microbial diversity present in each soil texture. Van Elsas et al., (2002) reported that different soil management practices have a strong impact on soil bacterial and microbial populations, which can alter soil fertility levels and seedbank viability.

The influence of flooding period on Palmer amaranth seed damage is presented in Figure 3.4. Flooding periods of 4 and 5 months resulted in the greatest amount of damaged Palmer amaranth seeds ( $\mathrm{p}<0.0001)$ (Figure 3.4). Conversely, flooding period of 2 months resulted in the least amount of seed damage. Flooding for 1 month and 3 months resulted in more damaged seeds compared to 2 months. In addition, no-flooding resulted in similar levels of seed damage compared to flooding periods of 4 and 5 months. In this experiment, no-flooding was kept in the field for 5 months to evaluate the benefit of flooding for shorter periods compared to an extended no-flooding field condition. The amount of damaged seeds observed in no-flooding may be a result of exposure to adverse weather conditions throughout the experiment (Table 3.2). If noflooding had been removed earlier, less seed damage would be expected in no-flooding compared to flooding periods of 4 and 5 months. Although seed damage can be used as a parameter to estimate seed coat deterioration, further germination analysis is required to quantify seed viability.

\subsubsection{Total Palmer amaranth seed germination}

Analysis of fixed effects for total Palmer amaranth germination are presented in Table 3.3. Palmer amaranth seed germination did not differ due to soil texture $(\mathrm{p}=$ 0.1470). Although distinct seed characterization was previously reported, this did not translate into germination differences across soil textures. The abiotic and biotic factors 
that contributed to differences in seed characterization may not be impact embryo damage.

The interaction of flooding period and seed burial depth affected total Palmer amaranth seed germination $(\mathrm{p}<0.0001)$ (Table 3.3). Palmer amaranth seed germination was $23 \%$ greater when seeds were buried at $15 \mathrm{~cm}$ in no-flooding conditions compared to seeds placed on the soil surface (Table 3.4). Besides protecting seeds from damage caused by small insects, seed burial also reduces exposure to unfavorable environmental conditions, minimizing weathering and increasing seed viability (Leon et al., 2004; Forcella, 2003; Wijayratne and Pyke, 2012). Korres et al., (2018) reported increased Palmer amaranth and common waterhemp [Amaranthus tuberculatus (Moq.) J. D. Sauer] seed germination and longevity when seeds were buried at $17.5 \mathrm{~cm}$ compared to seeds placed at soil surface. Additionally, Sosnoskie et al., (2013) reported greater Palmer amaranth seed viability when seeds were buried at 10 and $40 \mathrm{~cm}$ compared to 1 and 2.5 cm burial depths. In contrast, results from this research show that seed burial depth did not increase Palmer amaranth germination in flooded conditions, regardless of flooding period (Table 3.4). The presence of water not only reduces light incidence, but it also negatively impacts oxygen availability, which is essential to growth and development of higher plants such as Palmer amaranth. Previous research reported that volumetric water content may be responsible for differences in Palmer amaranth seed persistence in different soils (Korres et al., 2018). Therefore, the advantageous effect of burial on seed germination was observed only in no-flooding treatments.

Flooding periods of 3, 4, and 5 months reduced Palmer amaranth seed germination compared to no-flooding at both burial depths (Table 3.4). Flooding periods 
of 3, 4, and 5 months reduced Palmer amaranth seed germination compared to noflooding by $10 \%$ ( 3 and 4 months) and $14 \%$ at $0 \mathrm{~cm}$ burial depth and $36 \%, 40 \%$, and $41 \%$ at $15 \mathrm{~cm}$ burial depth, respectively (Table 3.4). Extended periods of oxygen deficiency reduce seed germination and viability of Texasweed (Caperonia palustris L.) and barnyardgrass [Echinochloa crus-galli (L.) Beauv.] (Abouziena et al., 2015; Counce \& Nilda, 2006). Additionally, increased soil moisture conditions favor the establishment and colonization of saprophytic fungi and bacteria, which exercise an important role in seed mortality (Pakeman et al., 2012).

Palmer amaranth seed germination is strongly affected by edaphic conditions, especially under flooded conditions. These results indicate that fall-winter flooding can be used efficiently to improve Palmer amaranth control practices that utilize soil seedbank depletion as part of a total weed control management program.

\subsection{Conclusion}

This research demonstrates that flooding can be used to effectively reduce Palmer amaranth seed germination in sandy loam, silt, and silt loam soil textures. Flooding periods conducted for 3, 4, and 5 months resulted in the greatest reduction in Palmer amaranth seed germination. Additionally, seed burial depth did not increase Palmer amaranth seed germination under flooded conditions. Coupled with a sustainable and economically viable in-season weed control program, the use of flooding could be adopted as a reliable and effective practice to optimize Palmer amaranth soil seedbank depletion, especially in areas infested with herbicide-resistant biotypes. 
Table 3.1 Analysis of variance probability values for normal and damaged Palmer amaranth (AMAPA) seeds in 2016 and 2017.

\begin{tabular}{|c|c|}
\hline Fixed effects & Seed characterization (normal/damaged) \\
\hline & ---------------------p-values ${ }^{\mathrm{a}}$--------------------- \\
\hline Flooding period & $<0.0001$ \\
\hline Soil texture & 0.0419 \\
\hline Flooding period ${ }^{*}$ soil texture & 0.9993 \\
\hline Seed burial depth & 0.4303 \\
\hline Flooding period*seed burial depth & 0.0677 \\
\hline Soil texture* seed burial depth & 0.9520 \\
\hline $\begin{array}{l}\text { Flooding period*soil texture* seed burial } \\
\text { depth }\end{array}$ & 0.8182 \\
\hline
\end{tabular}

Table 3.2 Air temperature and precipitation averages in Starkville, MS during experiment duration in 2016 and 2017.

\begin{tabular}{lcc}
\hline Month & Air temperature & ${\text { Precipitation } \mathrm{mm}^{\mathrm{a}}}^{\mathrm{a}}$ \\
\hline & ${ }^{\circ} \mathrm{C}$ & $\mathrm{mm}$ \\
October & 20 & 28 \\
November & 14 & 58 \\
December & 8 & 128 \\
January & 7 & 93 \\
February & 12 & 174 \\
\hline
\end{tabular}

${ }^{a}$ Air temperature and precipitation were averaged over month and year. 
Table 3.3 Analysis of variance probability values for total Palmer amaranth (AMAPA) seed germination in 2016 and 2017.

\begin{tabular}{|c|c|}
\hline Fixed effects & Total AMAPA seed germination \\
\hline & --------------------p-values a \\
\hline Flooding period & $<0.0001$ \\
\hline Soil texture & 0.1470 \\
\hline Flooding period*soil texture & 0.9523 \\
\hline Seed burial depth & 0.5739 \\
\hline Flooding period*seed burial depth & $<0.0001$ \\
\hline Soil texture*seed burial depth & 0.5779 \\
\hline $\begin{array}{l}\text { Flooding period*soil texture* seed burial } \\
\text { depth }\end{array}$ & 0.9994 \\
\hline
\end{tabular}

Table 3.4 Total Palmer amaranth (AMAPA) seed germination as a result of flooding period and seed burial depth pooled across soil texture and year.

\begin{tabular}{lcc}
\hline Flooding period & Seed burial depth & $\begin{array}{c}\text { Total AMAPA seed } \\
\text { germination }\end{array}$ \\
\hline Months & $\mathrm{cm}$ & $\%$ \\
No-flooding & 0 & $21 \mathrm{~b}$ \\
1 & 15 & $44 \mathrm{a}$ \\
& 0 & $15 \mathrm{bc}$ \\
2 & 15 & $14 \mathrm{bcd}$ \\
& 0 & $14 \mathrm{bcd}$ \\
3 & 15 & $13 \mathrm{~cd}$ \\
4 & 0 & $11 \mathrm{~cd}$ \\
& 15 & $8 \mathrm{cde}$ \\
5 & 0 & $11 \mathrm{cde}$ \\
& 15 & $4 \mathrm{ef}$ \\
& 0 & $7 \mathrm{de}$ \\
${ }^{a}$ Means followed by the same letter are not significantly different according to Fisher's \\
protected LSD $(\alpha=0.05)$.
\end{tabular}




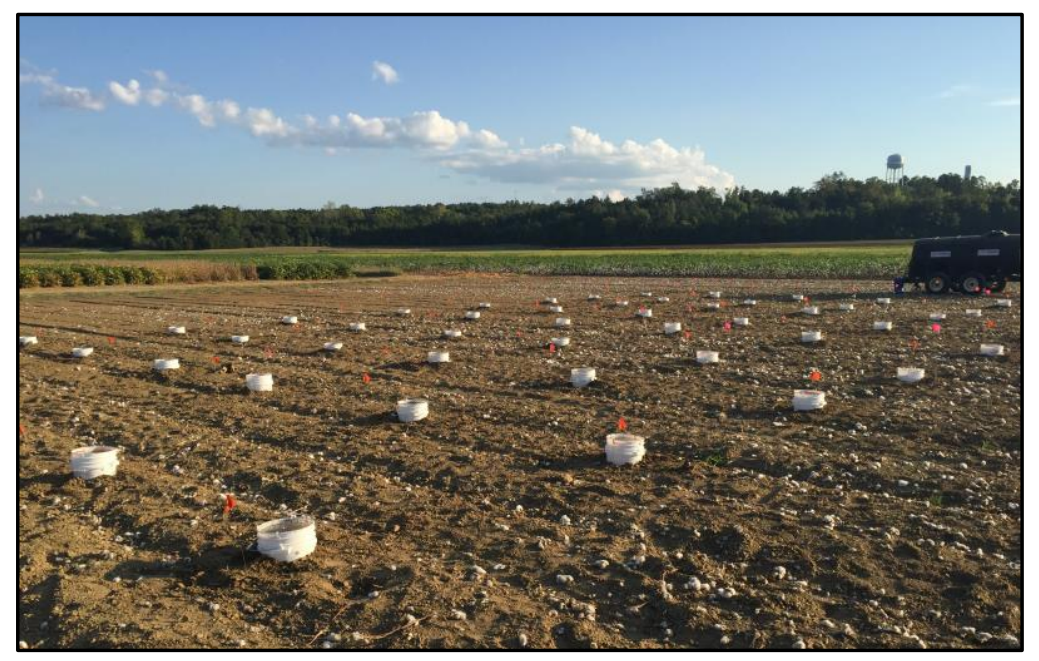

Figure 3.1 Experimental area at the R. R. Foil Plant Research Center in Starkville, MS.

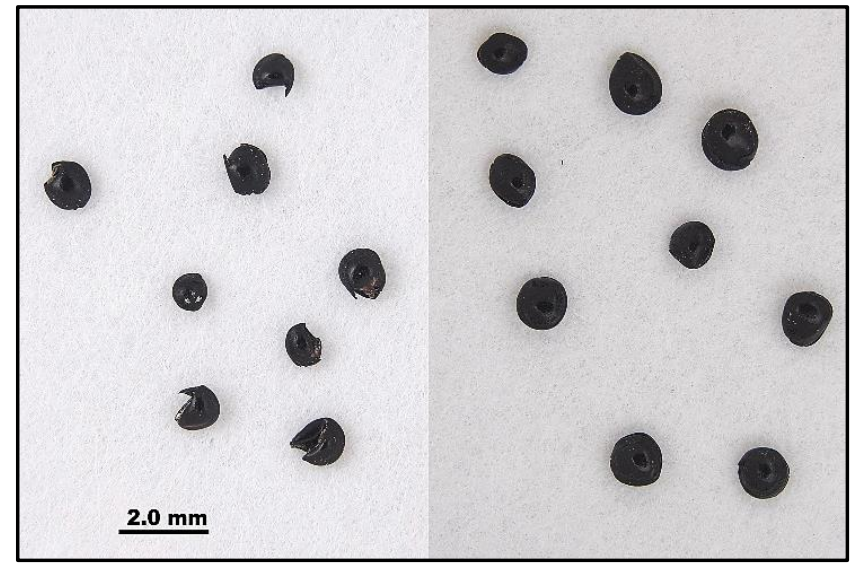

Figure 3.2 Visual aspects used for damaged (left) and normal (right) Palmer amaranth seed characterization. 


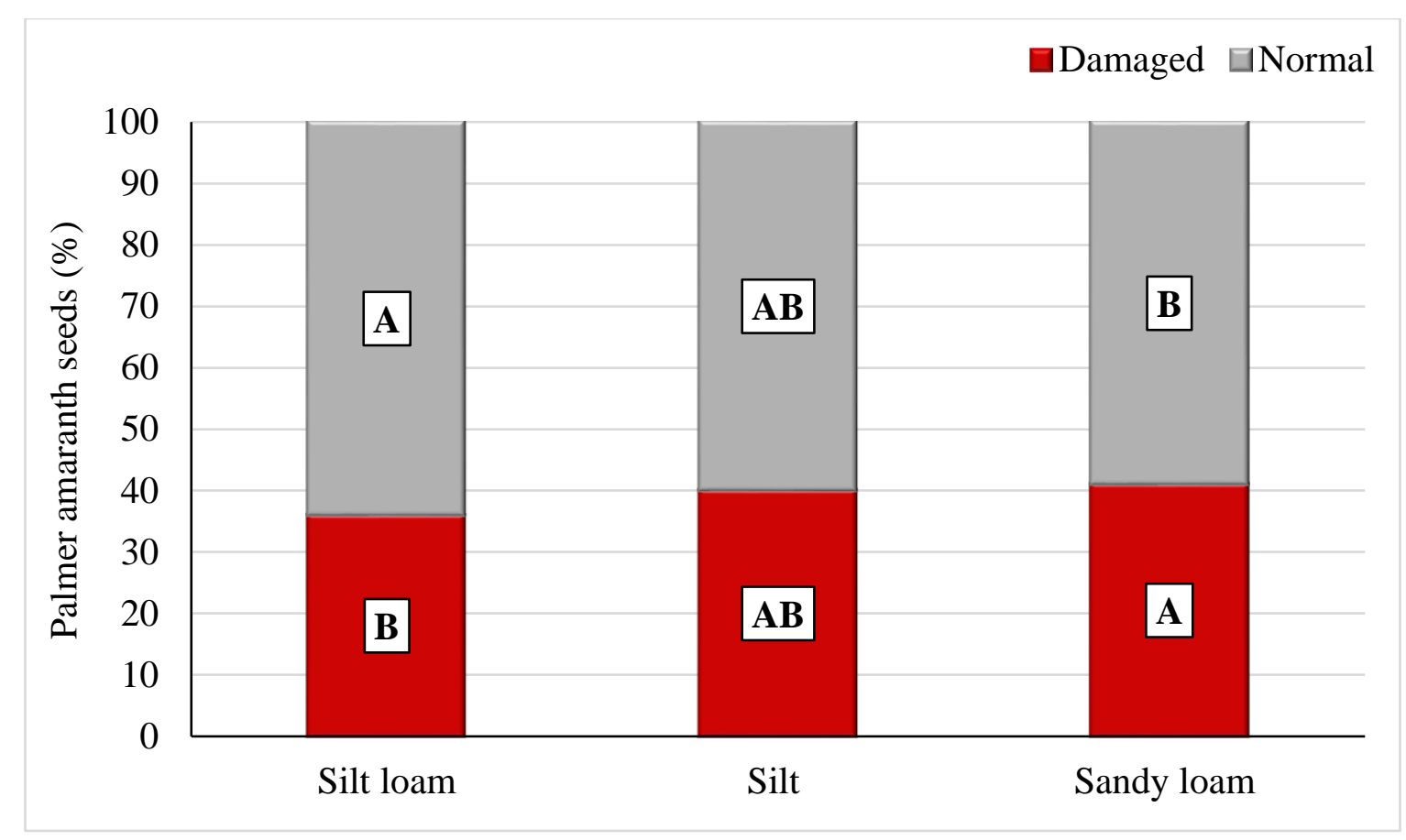

Figure 3.3 Palmer amaranth (AMAPA) seed characterization based on soil texture in 2016 and 2017.

Means within the same color followed by the same letter are not significantly different according to Fisher's Protected LSD $(\alpha=0.05)$. 


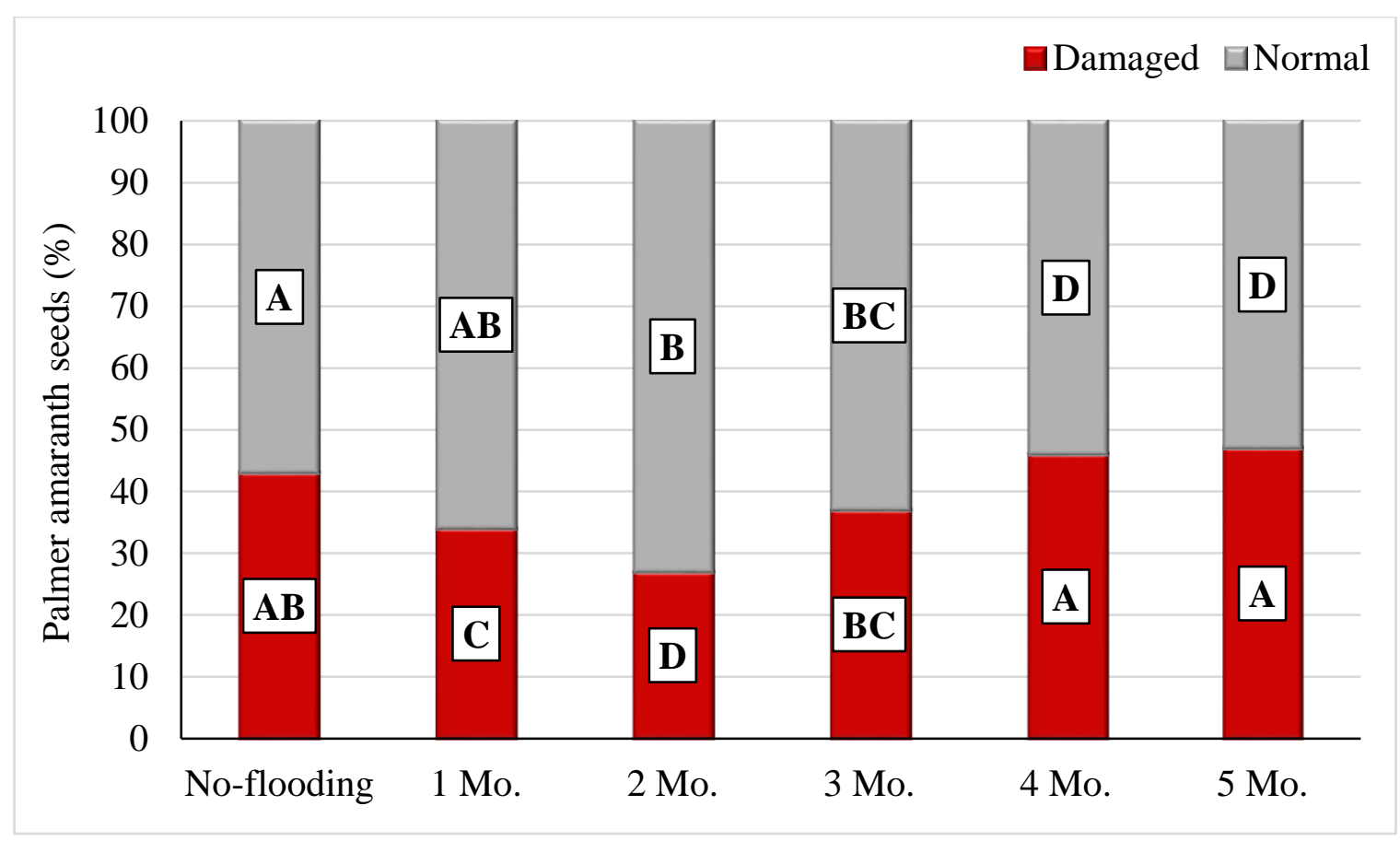

Figure 3.4 Palmer amaranth (AMAPA) seed characterization in response to flooding period pooled across seed burial depth, soil texture, and year.

Means within the same color followed by the same letter are not significantly different according to Fisher's Protected LSD $(\alpha=0.05)$. 


\subsection{Literature Cited}

Abouziena, H.F., El-Awadi, M.E., EL-Saeid, H.M., El-Metwally, I.M., El-Desouki, I.R., Singh, M. 2015. Influence of environmental factors, flooding period and seeding depth on germination and emergence of barnyardgrass [Echinochloa crus-galli (L.) Beauv.]. Int. J. Chemtech Res 8(6):459-467.

Baskin, J.M., Baskin, C.C. 1987. Temperature requirements for after-ripening in buried seeds of four summer annual weeds. Weed Res 27:397-402.

Baskin, J.M., Baskin, C.C. 2004. A classification system for seed dormancy. Seed Sci Res 14:1-16.

Benech-Arnold, R.L., Sanchez, R.A., Forcella, F., Kruk, B.C., Ghersa, C.M. 2000. Environmental control of dormancy in weed seed banks in soil. Field Crop Res 67(2):105-122.

Bond, J.A., Oliver, L.R., Stephenson, D.O. 2006. Response of Palmer amaranth (Amaranthus palmeri) accessions to glyphosate, fomesafen, and pyrithiobac. Weed Technol 20(4):885-892.

Burke, I.C., Schroeder, M., Thomas, W.E., Wilcut, J.W. 2007. Palmer amaranth interference and seed production in peanut. Weed Technol 21(2):367-371.

Chauhan, B.S., Johnson, D.E. 2009. Germination ecology of spiny (Amaranthus spinosus) and slender amaranth (A. viridis): troublesome weeds of direct-seeded rice. Weed Sci 4:379-385.

Costea, M.S., Weaver, S.E., Tardif, F.J. 2004. The biology of Canadian weeds. Can J Plant Sci 84:631-668.

Counce, P.A., Burgos, N.R. 2006. Agronomic practices in addition to herbicides for improving weed control in dry-seeded flooded rice. J Sustain Agric 28(3):145156.

Ehleringer, J. 1985. Annuals and perennials of warm deserts. Physiological Ecology of North American Plant Communities 162-180.

Emory, J. 1994. Just add water. J Nat Conserv 44:11-15.

Finch-Savage, W.E., Leubner-Metzger, G. 2006. Seed dormancy and the control of germination. New Phytol 171:501-523.

Forcella, F. 2003. Debiting the seedbank: priorities and predictions. Aspects Appl Biol 69:151-162. 
Forcella, F., Arnold, R.L.B., Sanchez, R., Ghersa, C.M. 2000. Modeling seedling emergence. Field Crops Res 67(2):123-139.

Gallagher, R.S., Cardina, J. 1998. Phytochrome-mediated Amaranthus germination I: effect of seed burial and germination temperature. Weed Sci 48-52.

Graeber, K.A.I., Nakabayashi, K., Miatton, E., Leubner-Metzger, G.E., Rhard and Soppe, W.J. 2012. Molecular mechanisms of seed dormancy. Plant Cell Environ $35: 1769-1786$.

Guo P., Al-Khatib, K. 2003. Temperature effects on germination of redroot pigweed (Amaranthus retroflexus), Palmer amaranth (A. palmeri), and common waterhemp (A. rudis). Weed Sci 51:869-875.

Hardke, J., Schmidt, L., Mazzanti, R. 2013. Arkansas rice quick facts. University of Arkansas Division of Agriculture, Research and Extension. http://www.agriculture.org/crops/rice/quick_facts/2013_rice_quick_facts.pdf (Accessed 10 Feb. 2017).

Jha, A.K., Dwivedi, B.S., Shrivastava, A.K., Soni, M., Taniwaki, K., Kokuryu, T., Kitagawa, I. Tateishi, K., Tsuji, K, and Kobayashi, S. 2014. Effect of drainage, tillage, and land configurations on root, nodules, and yield of soybean in vertisols. Soybean Res 194.

Keeley, P.E., Carter, C.H., Thullen, R.J. 1987. Influence of planting date on growth of Palmer amaranth (Amaranthus palmeri). Weed Sci 35:199-204.

Korres, N.E., Norsworthy, J.K., Young, B.G., Reynolds, D.B., Johnson, W.G., Conley, S.P., Smeda, R.J., Mueller, T.C., Spaunhorst, D.J., Gage, K.L. and Loux, M. 2018. Seedbank persistence of Palmer amaranth (Amaranthus palmeri) and waterhemp (Amaranthus tuberculatus) across diverse geographical regions in the United States. Weed Sci 66(4):446-456.

Leon, R.G., Bassham, D.C., Owen, M.D. 2006. Germination and proteome analyses reveal intraspecific variation in seed dormancy regulation in common waterhemp (Amaranthus tuberculatus). Weed Sci 54:305-315.

Leon, R.G., Knapp, A.D., Owen, M. D. K. 2004. Effect of temperature on germination of common waterhemp (Amaranthus tuberculatus), giant foxtail (Setaria faberi), and velvetleaf (Abutilon theophrasti). Weed Sci 52(1):67-73.

Manley, S.W. 1999. Ecological and agricultural values of winter-flooded rice fields in Mississippi (Doctoral dissertation, Mississippi State University. Department of Wildlife and Fisheries). 
Manley, S.W., Kaminski, R.M., Reinecke, K.J., Gerard, P.D. 2005. Agronomic implications of waterfowl management in Mississippi rice fields. Wildlife Society Bulletin 33:981-992.

Moldenhauer, K., Slaton, N. 2001. Rice growth and development. Rice production handbook. 7-14.

Muzzi, D. 1994. Winter floods offer duck habitat and crop savings. Rice Journal 97(6):89.

Norsworthy, J.K., Griffith, G., Griffin, T., Bagavathiannan, M., Gbur, E.E. 2014. In-field movement of glyphosate-resistant Palmer amaranth (Amaranthus palmeri) and its impact on cotton lint yield: evidence supporting a zero-threshold strategy. Weed Sci 62(2):237-249.

Omani, E.N., Haigh, A.M., Medd, R.W., Nicol, H.I. 1999. Changes in germinability, dormancy and viability of Amaranthus retroflexus as affected by a depth and duration of burial. Weed Res 39:345-354.

Pakeman, R.J., Small, J.L., Torvell, L. 2012. Edaphic factors influence the longevity of seeds in the soil. Plant Ecology, 213(1), pp.57-65.

Pons, T.L. 1991. Induction of dark dormancy in seeds: its importance for the seed bank in the soil. Funct Ecol 5:669-675.

Sauer, J. D. 1955. Revision of the dioecious amaranths. Madrono 13:5-46.

Sauer, J.1957. Recent migration and evolution of the dioecious amaranths. Evolution 11:11-31.

Sosnoskie, L.M., Webster, T.M., and Culpepper, A.S. 2013. Glyphosate resistance does not affect Palmer amaranth (Amaranthus palmeri) seed bank longevity. Weed Sci 61(2):283-288.

Taylorson, R.B., Borthwick, H.A. 1969. Light infiltration by foliar canopies: significance for light-controlled weed seed germination. Weed Sci 17:48-51.

Tolk, J.A., Howell, T.A., Evett, S.R. 1999. Effect of mulch, irrigation, and soil type on water use and yield of maize. Soil Till Res 50(2):137-147.

Van Elsas, J.D., Garbeva, P., Salles, J. 2002. Effects of agronomical measures on the microbial diversity of soils as related to the suppression of soil-borne plant pathogens. Biodegradation, 13(1):29-40. 
Webster, T.M., Grey, T.L. 2015. Glyphosate-resistant Palmer amaranth (Amaranthus palmeri) morphology, growth, and seed production in Georgia. Weed Sci 63:264272.

Wijayratne, U.C., Pyke, D.A. 2012. Burial increases seed longevity of two Artemisia tridentata (Asteraceae) subspecies. Am J Bot 99:438-447. 
CHAPTER IV

\author{
EFFECT OF NITROGEN FERTILIZER APPLICATION RATE ON TARNISHED \\ PLANT BUG (LYGUS LINEOLARIS PALISOT DE BEAUVOIS) \\ INFESTATION IN PALMER AMARANTH \\ (AMARANTHUS PALMERI S. WATS.)
}

\title{
4.1 Abstract
}

Nitrogen is essential for plant vegetative growth and maturity. Different nitrogen fertilizer application rates may impact the relationship between plant hosts and insects. This research was conducted to determine if different nitrogen fertilizer application rates impacted the attractiveness of Palmer amaranth (Amaranthus palmeri S. Wats.) to tarnished plant bug (Lygus lineolaris Palisot de Beauvois). Nitrogen fertilizer rate did not influence tarnished plant bug population density. Furthermore, nitrogen fertilizer application rate resulted in different responses between years with respect to cumulative tarnished plant bug density at the end of peak migration. Data indicates that in 2016 and 2017, tarnished plant bug population density was highly affected by sampling date, but not by nitrogen fertilizer application rate. Conversely, nitrogen fertilizer application rate influenced cumulative tarnished plant bug density in 2018 , with $179 \mathrm{~kg} \mathrm{~N} \mathrm{ha}^{-1}$ resulting in the greatest number of insects. The interaction between tarnished plant bug population density and Palmer amaranth as influenced by nitrogen fertilizer application rate is not consistent across years which could be due to a wide array of biotic and abiotic factors. 
Further investigation is necessary to understand the complexity of these relationships and better address the impact of nitrogen rate on tarnished plant bug density in Palmer amaranth.

\subsection{Introduction}

Tarnished plant bug (Lygus lineolaris Palisot de Beauvois) (Hemiptera: Miridae) is a pest native to the eastern United States but has spread throughout extensive areas in North America (Walgenbach, 2015). Adults can damage several important agronomic crops such as apple (Malus pumila Mill.), cotton (Gossypium hirsutum L.), soybean [Glycine max (L.) Merr.], peaches [Prunus persica (L.) Batsch.], strawberry (Fragaria ananassa Duch.), and tomatoes (Solanum lycopersicum L.), and are 6 to $6.5 \mathrm{~mm}$ long, oval shaped, and somewhat flattened (Young, 1986; Spangler et al., 1991). Adults are normally brown in color, with reddish brown markings on the wings, but are distinguished by a small yellow-stripped triangle in the center of their back, which is denominated scutellum. In cotton, tarnished plant bugs prefer feeding on small to medium sized flower buds (squares) compared to other plant structures (Tugwell et al., 1976). The presence of a yellow stain in the outer surface of the developing cotton square indicates presence and feeding activity in a given area which generally results in abscission of small squares, leading to direct yield losses (Layton, 2000). In addition, tarnished plant bug damage to older squares results in abnormal flowers that, depending on the damage level, may limit pollination, resulting in abnormal bolls that often abscise (Pack and Tugwell, 1976).

Tarnished plant bug is considered the most economically important insect pest of cotton in the midsouthern United States (Musser et al., 2009; Cook, 2018). During the 
2017 growing season, the Lygus complex caused more fruit abscission than any other pest and tarnished plant bug alone infested more than 250,000 hectares resulting in loss of more than 100,000 cotton bales (Cook, 2018). In Mississippi, growers have averaged at least six insecticide applications for tarnished plant bug control since 2013 (Wood et al., 2016). Growers spend nearly US $\$ 277$ per hectare on tarnished plant bug control which, combined with other input production costs make cotton less profitable compared to alternative crops (Wood et al., 2016). Costly insecticide applications can be attributed to the high levels of insecticide resistance in tarnished plant bug populations. Gore et al., (2012) reported that tarnished plant bug populations in Mississippi, Lousiana, and Arkansas have high levels of insecticide resistance. Resistance to pyrethroids, organophosphates, and carbamates were first reported in 1995, 2001, and 2007, respectively (Snodgrass, 1996; Snodgrass and Scott, 2000; Snodgrass et al., 2009). The current insecticide resistance scenario forces growers to heavily rely on neonicotinoid insecticide applications, increasing selection pressure for these insecticides. As resistant tarnished plant bug biotypes continue to spread, growers have become more concerned about the level of damage and control tactics that could be utilized to optimize the control of this pest. Given the increased difficulties in controlling tarnished plant bugs in cotton, alternative integrated pest management strategies are needed.

Typically, tarnished plant bugs are attracted by vigorous developing and vibrant cotton plants (Willers et al., 2000). In terms of cotton development and production, soil fertility is a dominating factor. Research conducted by Varco et al., (1999) reported that excessive nitrogen applications in cotton may result in increased vegetative growth leading to delayed maturity. Although differences in nitrogen application rate vary 
according to soil type, growing conditions, crop rotation, and management practices, an average of $135 \mathrm{~kg} \mathrm{~N} \mathrm{ha}^{-1}$ to $157 \mathrm{~kg} \mathrm{~N} \mathrm{ha}^{-1}$ of cotton is applied in Mississippi (Dodds, 2018). Use of proper nitrogen fertilizer application rate associated with early planting date and early maturity varieties may significantly reduce the number of insecticide applications required for tarnished plant bug control (Adams et al., 2013).

Tarnished plant bug overwinters and can be found in numerous weeds, vegetables, and fruits. In total, tarnished plant bug utilizes more than 300 plant species as hosts, and 169 of these species have been reported in the Mississippi Alluvial Valley region (Sudbrink et al., 2015). Palmer amaranth (Amaranthus palmeri S. Wats.) is a host for tarnished plant bug, especially in agricultural areas (Chahal et al., 2015). Snodgrass (2003) reported that tarnished plant bug migrates from cotton back to wild hosts in the fall, where adults in diapause are produced to overwinter. Tarnished plant bug is attracted by food resources present on reproductive structures, especially pollen. Palmer amaranth flowers are small (2 to $3.5 \mathrm{~mm}$ ) and clustered together to form terminal cylindrical inflorescences that can spike up to $60 \mathrm{~cm}$ long from the central stem; inflorescences in lateral branches are similar in structure but smaller in length (Ward et al., 2013). The best way to distinguish male and female flowers is by touch; male inflorescences are softer, while female inflorescences feel spikier because of the pointy bracts. Each female plant is capable of producing up to 600,000 seeds (Keeley et al., 1987). Thus, being able to provide a considerable amount of food resources to insects that use this species as a host.

The influence of nitrogen rate on Palmer amaranth attractiveness to tarnished plant bug has not been documented in Mississippi. Therefore, the objective of this 
experiment was to evaluate different nitrogen fertilizer application rates on tarnished plant bug infestation density with Palmer amaranth as a host.

\subsection{Materials and Methods}

Experiments were conducted at the Hood Farms in Dundee, MS (34 32' $39^{\prime \prime}$ N; $90^{\circ} 28^{\prime} 22^{\prime}$ W) on a Sharkey clay soil in 2016,2017 , and 2018. Studies were conducted in an area with high density of indigenous Palmer amaranth infestation. Prior to study initiation, a burndown application of paraquat (Gramoxone ${ }^{\circledR}$ SL, Syngenta Crop Protection LLC, Greensboro, NC 27409) at $0.56 \mathrm{~kg}$ ai ha ${ }^{-1}$ was applied using a $\mathrm{CO}_{2^{-}}$ propelled backpack sprayer equipped with TeeJet XR 110015 nozzles $\left(\right.$ TeeJet $^{\circledR}$ Technologies, Springfield, IL 62703) at $276 \mathrm{kPa}$ pressure to eliminate weeds present in the area and favor Palmer amaranth emergence. Nitrogen fertilizer applied was 32\% (320-0) urea ammonium nitrate (UAN) in 2016, and 30-0-0-2.5 S in 2017 and 2018.

Nitrogen fertilizer was applied using a tractor mounted with a 4-row knife applicator (Short Line MFG, Shaw, MS 38773) equipped with flow rate controller when Palmer plants were 5 to $10 \mathrm{~cm}$ in height. Treatments consisted of nitrogen fertilizer applied at 45 , 90, 135, and $179 \mathrm{~kg} \mathrm{~N} \mathrm{ha}^{-1}$. A nontreated check was used for treatment comparison. Plots were 4 meters wide and 12 meters long. Treatments were arranged in a randomized complete block design with four replications. Nitrogen fertilizer application dates in 2016, 2017, and 2018 are presented in Table 4.1.

Tarnished plant bug infestation was collected weekly following nitrogen fertilizer application date until September in 2016-2017, and October in 2018 (Table 4.1). Differences in nitrogen fertilizer application dates were due to unfavorable weather conditions and equipment logistics. Tarnished plant bug densities were collected by 
taking 25 sweeps using a $38 \mathrm{~cm}$ diameter sweep net per plot. Samples were placed inside plastic bags and brought to the R. R. Foil Plant Science Research Center in Starkville, MS for tarnished plant bug enumeration under a microscope. At the end of the experiment, ten Palmer amaranth plants per plot were selected for plant height measurement and sex determination. Palmer amaranth infestation density was also determined using a $0.25 \mathrm{~m}^{2}$ quadrat. Data were subjected to analysis of variance using PROC MIXED procedure in SAS v.9.4 (SAS ${ }^{\circledR}$ Institute Inc., Cary, NC 27513) and means were separated using Fischer's protected LSD at 0.05 level of significance. Nitrogen fertilizer application rate, sampling week, and year were analyzed as fixed factors. Replication was added to the model as a random factor. Estimation of regression parameters (nitrogen fertilizer application rate and sampling week) for cumulative tarnished plant bug infestation was conducted for each year in SAS v.9.4 using PROC GLM procedure. Regression analyses were conducted using log-transformed cumulative density during the highest peak of infestation (July and August).

\subsection{Results and Discussion}

Cumulative tarnished plant bug population densities were calculated for each nitrogen fertilizer application rate to facilitate interpretation of long-term effects. For better comparison analysis, data from July and August were selected for estimation of cumulative tarnished plant bug population density in 2016, 2017, and 2018. The interaction between nitrogen fertilizer application rate and year was highly significant when analysis of variance was conducted across years, demonstrating that the same nitrogen fertilizer application rate had a different response with respect to cumulative tarnished plant bug density between years $(\mathrm{p}<0.0001)$ (Table 4.2). Similarly, the 
presence of an interaction between sampling week and year indicate differences in cumulative tarnished plant bug infestation in the same sampling week between years $(\mathrm{p}=$ 0.0460) (Table 4.2). Tarnished plant bug density estimations for each nitrogen fertilizer application rate in 2016, 2017, and 2018 are shown in Table 4.3. In 2016, Palmer amaranth plants that received nitrogen fertilizer at $90 \mathrm{~kg} \mathrm{ha}^{-1}$ had the largest cumulative number of tarnished plant bugs, followed by $45 \mathrm{~kg} \mathrm{ha}^{-1}$ and $179 \mathrm{~kg} \mathrm{ha}^{-1}$, respectively. However, results were not consistent in 2017. Nitrogen fertilizer application did not impact cumulative tarnished plant bug densities. Nontreated plots had greater cumulative tarnished plant bug infestation compared to plots that received nitrogen fertilizer. Conversely, nitrogen fertilizer applied at $179 \mathrm{~kg} \mathrm{ha}^{-1}$ and $90 \mathrm{~kg} \mathrm{ha}^{-1}$ resulted in the greatest cumulative tarnished plant bug infestation in 2018.

Total tarnished plant bug density was substantially lower in 2018 compared to previous years (Table 4.3.). Although nitrogen fertilizer application rate performed inconsistently across years, a decrease in tarnished plant bug population density was observed across nitrogen fertilizer application rate. Alterations in the number of available hosts in winter and spring, especially wild hosts found in marginal areas, ditches and road sides, and adoption of conservative management practices could have negatively impacted tarnished plant bug migration in 2018 (Zhu et al., 2004).

Sampling week had an impact on tarnished plant bug density $(\mathrm{p}<0.0001)$ (Table 4.2). Usually, migration occurs from May until August with peak migration in July (Snodgrass et al., 1984). The effect of nitrogen fertilizer application rate and sampling week as parameters for tarnished plant bug density were calculated using logtransformation of cumulative density during the highest peak of infestation. Therefore, 
analysis was performed considering July and August in each year. Estimation of regression parameters are shown in Table 4.4. In 2016 and 2017, differences in tarnished plant bug population densities were observed between sampling week other than nitrogen fertilizer application rate $(\mathrm{p}<0.0001)$. Figures 4.1 and 4.2 demonstrate changes in tarnished plant bug density over time and nitrogen fertilizer application rate in 2016 and 2017, respectively. The blue lines in each contour graph represent tarnished plant bug density. All nitrogen fertilizer application rates are found in close proximity to the same density line, which indicates the lack of correlation between these factors. By contrast, tarnished plant bug density was affected by nitrogen fertilizer application rate in 2018 (p $=0.0003)$. Nitrogen fertilizer applied at $179 \mathrm{~kg} \mathrm{ha}^{-1}$ resulted in the greatest tarnished plant bug density (Figure 4.3). Furthermore, Palmer amaranth plants were significantly taller when $179 \mathrm{~kg} \mathrm{ha}^{-1}$ of fertilizer was applied compared to 90 and $135 \mathrm{~kg} \mathrm{ha}^{-1}$ (Table 4.5). Keeley et al., (1987) reported that tall Palmer amaranth plants are more likely to produce greater number of reproductive structures. These factors could have contributed to the increased tarnished plant bug density where $179 \mathrm{~kg} \mathrm{~N} \mathrm{ha}^{-1}$ was applied in 2018. Palmer amaranth density and sex were not affected by nitrogen fertilizer application indicating that Palmer amaranth density and dioecism are not affected by nitrogen fertilizer application rate (Table 4.6).

\subsection{Conclusion}

Nitrogen fertilizer application rate does not consistently impact tarnished plant bug population densities on Palmer amaranth plants. Although the highest nitrogen fertilizer application rate resulted in the greatest cumulative tarnished plant bug density in 2018, results were not consistent in 2016 and 2017. Tarnished plant bug infestation 
density on Palmer amaranth was affected by sampling week other than nitrogen fertilizer application rate. Soil fertility as well as local insect and agronomic management practices could be responsible for the increased variability observed in this experiment. Overall, sampling week has a greater impact on tarnished plant bug infestation density on Palmer amaranth than nitrogen fertilizer application rate. 
Table 4.1 Nitrogen fertilizer application and sampling interval dates in Dundee, MS in 2016, 2017, and 2018.

\begin{tabular}{lcc}
\hline Year & $\begin{array}{c}\text { Nitrogen fertilizer } \\
\text { application date }\end{array}$ & Sampling interval \\
\hline 2016 & 06 June & 23 June - 02 Sep. \\
2017 & 01 July & 07 July -15 Sep. \\
2018 & 21 June & 29 Jun. - 05 Oct. \\
\hline
\end{tabular}

Table 4.2 Analysis of variance probability values for cumulative tarnished plant bug population density across years in Dundee, MS.

\begin{tabular}{|c|c|}
\hline Source of variation & Cumulative tarnished plant bug infestation \\
\hline & 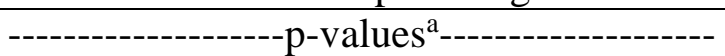 \\
\hline Nitrogen fertilizer rate & $<0.0001$ \\
\hline Week & $<0.0001$ \\
\hline Year & $<0.0001$ \\
\hline Nitrogen fertilizer rate* year & $<0.0001$ \\
\hline Week*year & 0.0460 \\
\hline
\end{tabular}

Probability values calculated based on data pooled across years. 
Table 4.3 Tarnished plant bug population density as influenced by nitrogen fertilizer rate in Dundee, MS in 2016, 2017, and 2018.

\begin{tabular}{lccc}
\hline & \multicolumn{3}{c}{ Cumulative tarnished plant bug population density ${ }^{\mathrm{a}, \mathrm{b}}$} \\
\hline Nitrogen fertilizer rate & 2016 & 2017 & 2018 \\
\hline $\mathrm{kg} \mathrm{ha}^{-1}$ & & & \\
Nontreated & $28 \mathrm{c}$ & $28 \mathrm{a}$ & $7 \mathrm{~b}$ \\
45 & $43 \mathrm{~b}$ & $19 \mathrm{c}$ & $6 \mathrm{~b}$ \\
90 & $52 \mathrm{a}$ & $24 \mathrm{abc}$ & $8 \mathrm{ab}$ \\
135 & $29 \mathrm{c}$ & $22 \mathrm{bc}$ & $4 \mathrm{~b}$ \\
179 & $45 \mathrm{ab}$ & $25 \mathrm{ab}$ & $10 \mathrm{a}$ \\
\hline
\end{tabular}

a Analysis of variance (ANOVA) conducted using data from July and August for each year independently.

${ }^{\mathrm{b}}$ Means within a column for each year followed by the same letter are not significantly different according to Fisher's protected LSD $(\alpha=0.05)$ 
Table 4.4 Regression of log cumulative tarnished plant bug population density with week and nitrogen fertilizer rate as source of variation in 2016, 2017 and 2018.

\begin{tabular}{lccc}
\hline Source of variation & Year & $R^{2}$ & Probability values \\
\hline Week & 2016 & 0.5914 & $<0.0001$ \\
Nitrogen Fertilizer rate & & & 0.3980 \\
Week*Nitrogen Fertilizer rate & & & 0.6896 \\
Week*Week & & 0.0077 \\
Nitrogen Fertilizer & & 0.5540 \\
rate*Nitrogen Fertilizer rate & & \\
& & \\
Week & 2017 & 0.2390 & $<0.0001$ \\
Nitrogen Fertilizer rate & & & 0.2865 \\
Week*Nitrogen Fertilizer rate & & & 0.2797 \\
Week*Week & & $<0.0001$ \\
Nitrogen Fertilizer & & 0.4293 \\
rate*Nitrogen Fertilizer rate & & & \\
& & \\
Week & 0.4836 & 0.0936 \\
Nitrogen Fertilizer rate & & & 0.0014 \\
Week*Nitrogen Fertilizer rate & & & 0.0003 \\
Week*Week & & 0.0811 \\
Nitrogen Fertilizer & & \\
rate*Nitrogen Fertilizer rate & & & 0.0006 \\
\hline
\end{tabular}

${ }^{\mathrm{a}}$ Linear and quadratic effect functions were analyzed for better trend identification. 
Table 4.5 Palmer amaranth (AMAPA) height, density, and sex probability values respective to nitrogen fertilizer application rate pooled across years.

\begin{tabular}{lc}
\hline Response variable & Probability values $^{\mathrm{a}}$ \\
\hline Plant height & 0.0486 \\
Plant density & 0.6006 \\
Plant sex & 0.2190 \\
\hline
\end{tabular}

${ }^{a}$ Probability values calculated based on data pooled across years.

Table 4.6 Palmer amaranth (AMAPA) height as influenced by nitrogen fertilizer application rate pooled across years.

\begin{tabular}{lc}
\hline Nitrogen fertilizer rate & AMAPA height $^{\mathrm{a}}$ \\
\hline $\mathrm{kg} \mathrm{ha}^{-1}$ & $\mathrm{~cm}$ \\
0 & $112 \mathrm{~b}$ \\
45 & $116 \mathrm{ab}$ \\
90 & $109 \mathrm{~b}$ \\
135 & $108 \mathrm{~b}$ \\
179 & $128 \mathrm{a}$ \\
\hline
\end{tabular}

${ }^{a}$ Probability values calculated based on data pooled across years. 


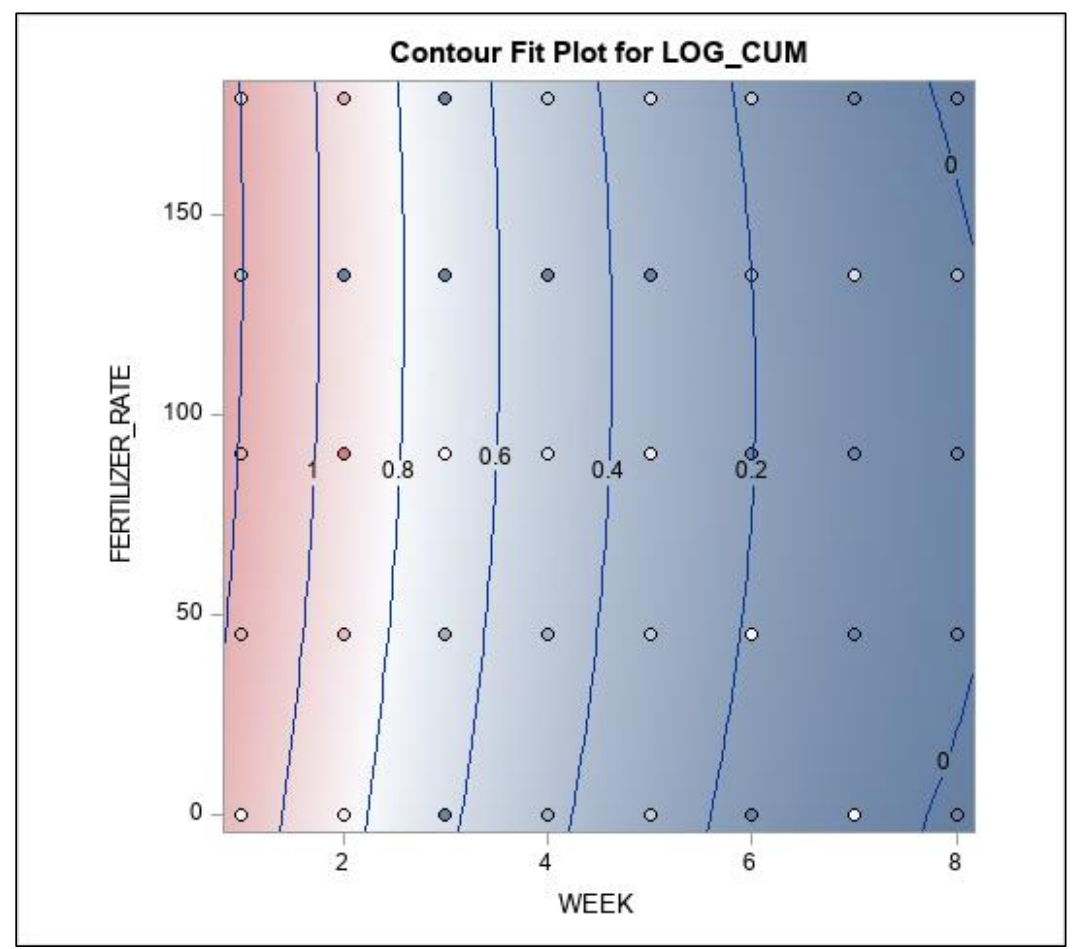

Figure 4.1 Contour graph for log-cumulative tarnished plant bug population density as influenced by nitrogen fertilizer application rate and sampling week in 2016. 


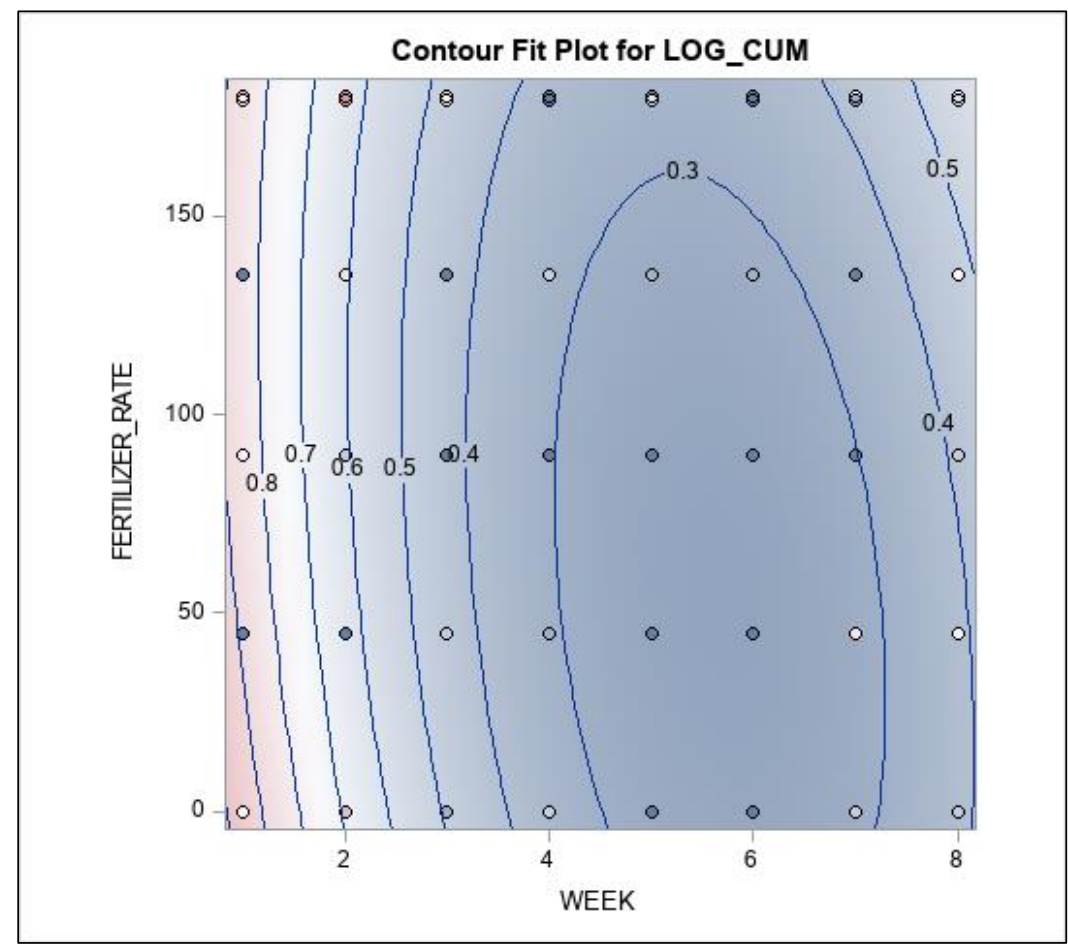

Figure 4.2 Contour graph for log-cumulative tarnished plant bug population density as influenced by nitrogen fertilizer application rate and sampling week in 2017. 


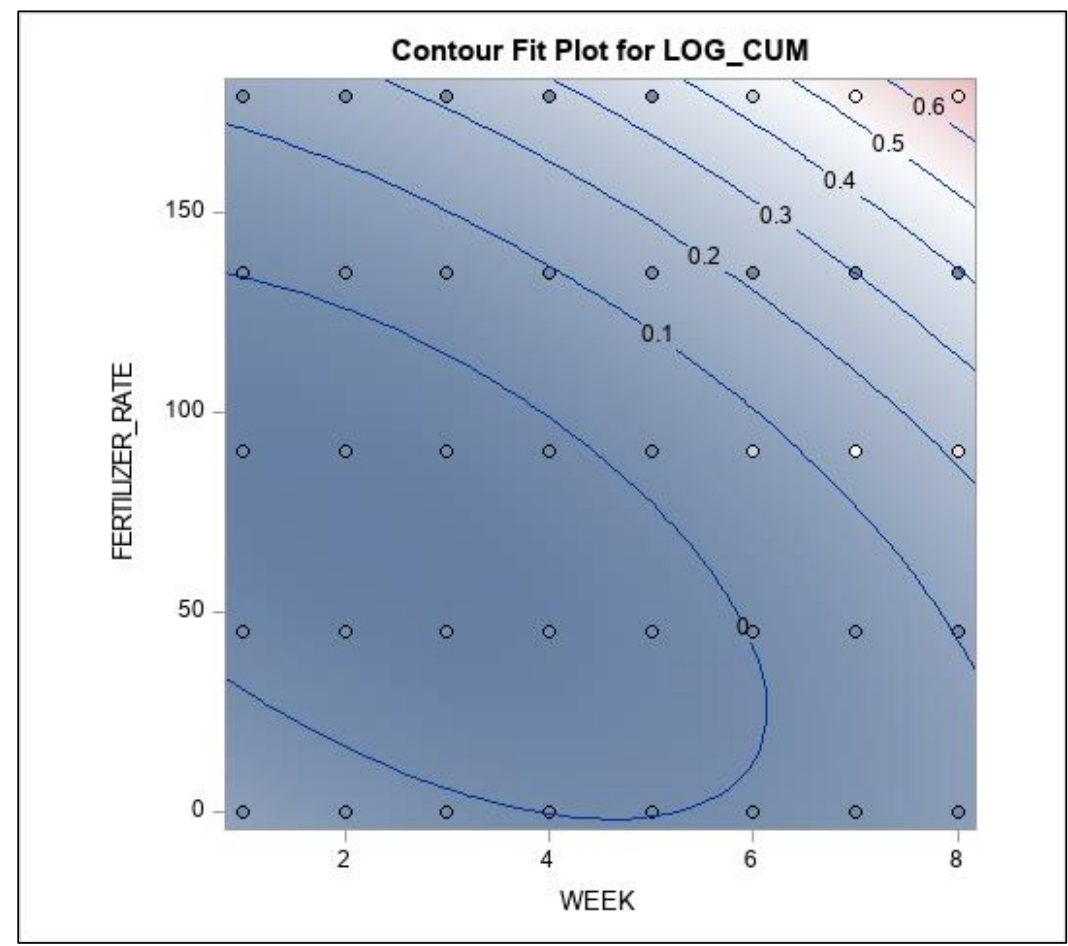

Figure 4.3 Contour graph for log-cumulative tarnished plant bug population density as influenced by nitrogen fertilizer application rate and sampling week in 2018. 


\subsection{Literature Cited}

Adams, B., Catchot, A., Gore, J., Cook, D., Musser, F. and Dodds, D. 2013. Impact of planting date and varietal maturity on tarnished plant bug (Hemiptera: Miridae) in cotton. J Econ Entomol 106(6):2378-2383.

Chahal, P.S., Aulakh, J.S., Jugulam, M., Jhala, A.J. 2015. Herbicide resistant Palmer amaranth (Amaranthus palmeri S. Wats.) in the United States: mechanisms of resistance, impact, and management. p. 1-29. in Price A, Kelton J, Sarunaite L eds. Herbicides, Agronomic Crops and Weed Biology. Rijeka, Croatia: InTech

Cook, D.R. 2018. Cotton insect losses 2017. p. 721-780. In Proceedings of the Beltwide Cotton Conferences, San Antonio, Texas. Memphis, TN: National Cotton Council of America; 3-5 January 2018.

Dodds, D.M. 2018. Nitrogen. Mississippi State University Ext. Serv. http://extension.msstate.edu/content/nitrogen/ (accessed 20 Jan. 2019).

Gore, J., Catchot, A., Musser, F., Greene, J., Leonard. B.R., Cook, D.R., Snodgrass, G.L., Jackson, R. 2012. Development of a plant-based threshold for tarnished plant bug (Hemiptera: Miridae) in cotton. J Econ Entomol 105:2007-2014.

Keeley, P.E., Carter, C.H., Thullen. R.J. 1987. Influence of planting date on growth of Palmer amaranth (Amaranthus palmeri). Weed Sci 35:199-204.

Layton, M.B. 2000. Biology and damage of the tarnished plant bug, Lygus lineolaris, in cotton. Southwest. Entomol (Suppl. 23):7-20.

Musser, F.R., Catchot, A.L., Stewart, S.D., Bagwell, R.D., Lorenz, G.M., Tindall K.V., Studebaker, G.E., Leonard, B.R., Akin, D.S., Cook, D.R., Daves, C.A. 2009. Tarnished plant bug (Hemiptera: Miridae) thresholds and sampling comparisons for flowering cotton in the midsouthern United States. J Econ Entomol 102:18271836 .

Pack, T.M., Tugwell, P. 1976. Clouded and tarnished plant bugs on cotton: a comparison of injury symptoms and damage on fruit parts. Agricultural Experiment Station, Division of Agriculture, University of Arkansas.

Snodgrass, G.L., Gore, J., Jackson, R., Abel, C.A. 2009. Acephate resistance in populations of the tarnished plant bug (Heteroptera: Miridae) from the Mississippi River Delta. J Econ Entomol 102:699-707.

Snodgrass, G.L., Scott, W.P. 2000. Seasonal changes in pyrethroid resistance in tarnished plant bug (Heteroptera: Miridae) populations during a three-year period in the Delta area of Arkansas, Louisiana, and Mississippi. J Econ Entomol 93:441-446. 
Snodgrass, G.L. 1996. Insecticide resistance in field populations of the tarnished plant bug (Heteroptera: Miridae) in cotton in the Mississippi Delta. J Econ Entomol 89: 783-790.

Snodgrass, G.L. 2003. Role of reproductive diapause in the adaption of the tarnished plant bug (Heteroptera: Miridae) to its winter habitat in the Mississippi River Delta. Environ Entomol 32:945-952.

Snodgrass, G.L., Scott, W.P., Smith, J.W. 1984. Host plants and seasonal distribution of the tarnished plant bug (Hemiptera: Miridae) in the Delta of Arkansas, Louisiana, and Mississippi. Environ Entomol 13:110-116.

Spangler, S., Weires, R., Agnello, A. 1991. Insect Identification Sheet No.21, Tarnished Plant Bug Lygus lineolaris. http://www.nysae.cornell.edu/ipmet/ny/friuts/FruitFS/tarnishplantbug.html (accessed 14 Jan. 2018)

Sudbrink, D.L., Thomson, S.J., Fletcher, R.S., Harris, F.A., English, P.J. and Robbins, J.T. 2015. Remote sensing of selected winter and spring host plants of tarnished plant bug (Heteroptera: Miridae) and herbicide use strategies as a management tactic. American J Plant Sci 6(8):1313.

Tugwell, P., Young, S.C., Dumas, B.A., Phillips, J.R. 1976. Plant bugs in cotton: importance of infestation time, types of cotton injury, and significance of wild hosts near cotton, Arkansas Agricultural Experiment Station Report. Report Series 227, University of Arkansas, Fayetteville, AR.

Varco, J.J., Spurlock, S.R., Sanabria-Garro, O.R. 1999. Profitability and nitrogen rate optimization associated with winter cover management in no-tillage cotton. J Prod Ag 12:91-95.

Walgenbach J. 2015. Tarnished Plant Bug. North Carolina State University Cooperative Extension Resources. Cooperative Extension. https://content.ces.ncsu.edu/tarnished-plant-bug (accessed 14 Jan. 2018).

Ward, S.M., Webster, T.M., Steckel, L.E. 2013. Palmer amaranth (Amaranthus palmeri): a review. Weed Technol 27(1):12-27.

Wood, W., Gore, J., Catchot, A., Cook, D., Dodds, D., Krutz, L.J. 2016. Susceptibility of Flowering Cotton to Damage and Yield Loss from Tarnished Plant Bug (Hemiptera: Miridae). J Econ Entomol 109(3):1188-1195.

Young, O.P. 1986. Host plants of the tarnished plant bug, Lygus lineolaris (Heteroptera: Miridae). Ann Entomol Soc Am 79:747-762. 
Zhu, Y.C., Snodgrass, G.L. and Chen, M.S. 2004. Enhanced esterase gene expression and activity in a malathion-resistant strain of the tarnished plant bug, Lygus lineolaris. Insect Biochem Mol Biol 34(11):1175-1186. 


\section{APPENDIX A}

VISUAL PALMER AMARANTH (AMARANTHUS PALMERI S. WATS.) CONTROL AS INFLUENCED BY INTERACTION DROPLET SIZE AND LOCATION INTERACTION 
Table A.1 Visual Palmer amaranth control by site-year at 7 DAT following acifluorfen application.

\begin{tabular}{|c|c|c|c|}
\hline Location & Year & Droplet size & Control $^{\mathrm{a}, \mathrm{b}}$ \\
\hline & & $\mu \mathrm{m}$ & $\%$ \\
\hline \multirow[t]{18}{*}{ Dundee, MS } & 2016 & 150 & $46 \mathrm{ab}$ \\
\hline & & 300 & $75 \mathrm{a}$ \\
\hline & & 450 & $33 \mathrm{~b}$ \\
\hline & & 600 & $62 \mathrm{ab}$ \\
\hline & & 750 & $59 a b$ \\
\hline & & 900 & $52 \mathrm{ab}$ \\
\hline & 2017 & 150 & $53 \mathrm{~b}$ \\
\hline & & 300 & $84 \mathrm{a}$ \\
\hline & & 450 & $79 a$ \\
\hline & & 600 & $73 \mathrm{a}$ \\
\hline & & 750 & $74 \mathrm{a}$ \\
\hline & & 900 & $82 \mathrm{a}$ \\
\hline & 2018 & 150 & $59 \mathrm{ab}$ \\
\hline & & 300 & $70 \mathrm{a}$ \\
\hline & & 450 & $49 \mathrm{~b}$ \\
\hline & & 600 & $48 \mathrm{~b}$ \\
\hline & & 750 & $48 \mathrm{~b}$ \\
\hline & & 900 & $67 \mathrm{ab}$ \\
\hline \multirow[t]{12}{*}{ Beaver City, NE } & 2016 & 150 & $74 \mathrm{a}$ \\
\hline & & 300 & $69 \mathrm{ab}$ \\
\hline & & 450 & $41 \mathrm{~d}$ \\
\hline & & 600 & $64 \mathrm{~b}$ \\
\hline & & 750 & $51 \mathrm{c}$ \\
\hline & & 900 & $55 \mathrm{c}$ \\
\hline & 2017 & 150 & $90 \mathrm{a}$ \\
\hline & & 300 & $85 \mathrm{a}$ \\
\hline & & 450 & $83 a$ \\
\hline & & 600 & $80 a$ \\
\hline & & 750 & $83 \mathrm{a}$ \\
\hline & & 900 & $81 \mathrm{a}$ \\
\hline \multirow[t]{6}{*}{ Robinsonville, MS } & 2017 & 150 & $69 \mathrm{~b}$ \\
\hline & & 300 & $76 \mathrm{ab}$ \\
\hline & & 450 & $87 \mathrm{a}$ \\
\hline & & 600 & $76 \mathrm{ab}$ \\
\hline & & 750 & $75 \mathrm{ab}$ \\
\hline & & 900 & $71 \mathrm{~b}$ \\
\hline \multirow[t]{4}{*}{ Robinsonville, MS } & 2018 & 150 & $45 \mathrm{c}$ \\
\hline & & 300 & $74 \mathrm{a}$ \\
\hline & & 450 & $66 a b$ \\
\hline & & 600 & $58 \mathrm{abc}$ \\
\hline
\end{tabular}


Table A.1 (continued)

$65 a b$

900

$54 \mathrm{bc}$

${ }^{\mathrm{a}}$ Visual Palmer amaranth control was analyzed within each site-year.

${ }^{\mathrm{b}}$ Means within the site-year followed by the same letter are not significantly different according to Fisher's protected LSD $(\alpha=0.05)$. 